\title{
THE GLOBULAR CLUSTER SYSTEMS OF THREE VIRGO ELLIPTICALS
}

\author{
JUDITH G. COHEN \\ Palomar Observatory, California Institute of Technology, Pasadena, California 91125 \\ Received 25 June 1987; revised 12 November 1987
}

\begin{abstract}
An analysis of the systems of globular clusters around three of the brightest elliptical galaxies in the Virgo cluster (NGC 4406, NCG 4472, and NGC 4486) has been carried out with a digital detector whose field is 9 arcmin on a side. The point-source detections reach to $g=25 \mathrm{mag}$, and nearly all galaxies brighter than $g \approx 22.5 \mathrm{mag}$ can be eliminated. We find that the colors of the globular clusters show no detectable spatial gradient, and that the median color and the distribution in color are independent of cluster luminosity and spatial position within the halo of the underlying elliptical galaxy. It is, however, true that the median color of the globular clusters in each galaxy is at each point bluer than the light of the underlying halo. The median color of the Virgo globular systems is slightly redder than that of the set of globulars in the Milky Way, implying a mean metallicity difference of about a factor of 3. The magnitude distribution of the globular cluster system may be somewhat broader in NGC 4486 than it is in NGC 4472. The distribution is not a power law, having a definite turnover at $g \approx 24.2 \mathrm{mag}$, which constrains the distance to the Virgo cluster if one believes in a universal globular cluster luminosity function. In each of the three cases, the globular cluster system has the same ellipticity and position angle as the light of the halo of the underlying galaxy, but is more extended spatially in the case of NGC 4472 and NGC 4486.
\end{abstract}

\section{INTRODUCTION}

The extensive swarms of globular clusters around the brighter ellipticals in the Virgo cluster are objects of great interest as they may convey important information on galaxy formation and interactions between galaxies. This paper presents an analysis of the properties of the system of globular clusters around NGC 4406, NGC 4472, and NGC 4486, three of the brightest ellipticals in the Virgo cluster. A recent review by Harris (1986) summarizes the known properties of the globular cluster systems around several galaxies beyond the Local Group. The impressive ensemble surrounding NGC 4486 (also known as M87) has been studied by many astronomers, including Racine (1968), Ables, Newell, and O'Neill (1974), Harris and Smith (1976), Hanes (1977), Strom et al. (1981), van den Bergh, Pritchet, and Grillmair (1985), Grillmair, Pritchet, and van den Bergh (1986), and Lauer and Kormendy (1986). A preliminary reconnaissance of the globular cluster system of NGC 4472 (also known as M49) was given by Harris and Petrie (1978) and is updated in Harris (1986). Forte, Strom, and Strom (1981) present mean colors for the globular cluster systems around three other bright Virgo ellipticals. The present study offers a significant improvement over these earlier works, as it combines the advantages of a relatively large area surveyed to a deep magnitude limit and the use of a digital detector, which makes removal of the background light from the halo of the underlying galaxy easier and more accurate.

After a brief review of the observational material, which is identical to that of Cohen (1986), where the properties of the integrated light of the three galaxies were studied, we describe in Sec. II the assembly of the list of point sources and galaxies in the fields of the three Virgo ellipticals. The procedures used for the photometry are also indicated in Sec. II $b$, while the definition and properties of the background of galactic stars, foreground and background galaxies, quasars, etc., not associated with the systems of globular clusters around the Virgo ellipticals are outlined in Sec. III. Section IV presents the magnitude distribution of the globular clus- ter systems for each of the three galaxies and discusses various aspects of their completeness. The colors of the ensemble of globular clusters are discussed in the next section, while Sec. VI describes their spatial distribution. A summary of important results is given in Sec. VII, where our findings are compared and contrasted to those of the previous investigations listed above. The implications of these results for theories of galaxy formation are also outlined at the end of the final section. An Appendix describes the properties of the dwarf galaxies seen in our data which we believe to be associated with the Virgo cluster.

\section{ASSEMBLY OF THE LISTS OF CLUSTERS AND THEIR PROPERTIES \\ a) Finding the Clusters}

The observational data used here were described in Paper I. To repeat briefly, many $800 \mathrm{~s}$ exposures of each of the three galaxies were taken through the $g$ filter of the Thuan-Gunn system using the 4-Shooter at the Cassegrain focus of the $5 \mathrm{~m}$ Hale telescope at Palomar Mountain in March 1985. There is also one frame for each galaxy through the $r$ and the $i$ filter of the same photometric system with exposure times of 300 and $500 \mathrm{~s}$, respectively. As the scale is $0.334 \mathrm{arcsec} / \mathrm{pixel}$, each galaxy image is $\approx 9$ arcmin on a side, and is composed of four $800 \times 800$ pixel CCD frames $\approx 270$ arcsec on a side. The complete set of $g$ frames for each galaxy was inspected and the best frames were selected for further study. These were chosen on the basis of best transparency, darkest sky, and best seeing. (The typical FWHM of the image of a point source on these frames was three pixels or 1.0 arcsec.) For NGC 4472 and NGC 4486, seven extremely good frames were identified, while for NGC 4406 only six such frames were available. From each frame, the best-fit ellipses describing the background-galaxy light, as defined in Paper I, were subtracted, as was a constant representing the signal from the sky. The frames were then shifted by an integral number of pixels in the $x(\mathrm{E}-\mathrm{W})$ and $y(\mathrm{~N}-\mathrm{S})$ directions so as to coincide spatially, and were summed. (The maximum shift 
necessary for any of the frames was 12 pixels in $x$ and 16 pixels in $y$ ). (We subsequently denote the resulting summed frames as the MASTERG frames for each galaxy.) The typical FWHM of the image of a point source on the MASTERG frames was 1.1 arcsec.

The nucleus of the galaxy was in two cases deliberately offset from the center of the 4-Shooter image for reasons described in Paper I. The nuclei of NGC 4406, NGC 4472, and NGC 4486 were located ( $60 \operatorname{arcsec} E, 2 \operatorname{arcsec} S$ ), ( 50 $\operatorname{arcsec} \mathrm{W}, 8 \operatorname{arcsec} \mathrm{S})$, and ( $1 \operatorname{arcsec} \mathrm{E}, 3 \operatorname{arcsec} \mathrm{S})$ of the center of the 4-Shooter image, respectively. Thus, the nucleus of NGC 4406 was in chip 2 of the frames, that of NGC 4472 was in chip 3 , and that of NGC 4486 was approximately in the center of the total field.

A point-source detection code was then run over the MASTERG frames which finds crude (to within one pixel) object locations. This basically looked for sources with more than five continuous pixels higher than a threshold value, and no pixel higher than a maximum value. The choice of minimum value is not straightforward, as there were small residuals of the rather large subtracted background which varied spatially across the field. Thus, each of the four MASTERG frames for each galaxy was subdivided into up to 13 smaller regions within which the background could be considered as roughly constant. The minimum value for point-source detection was set at $200 \mathrm{DN}$ above the local background within each small region. For regions in the periphery of each galaxy, the rms dispersion about the local mean background was $85 \mathrm{DN}$. In practice, the total range over the entire chip of the variation in background (excluding the region within 50 arcsec of the nucleus of the galaxy) never exceeded $600 \mathrm{DN}$. (The electronics of the 4-Shooter are set so that $1 \mathrm{DN} /$ pixel $\approx 2$ electrons/pixel.) The maximum value was set as $n(0.9 \times \mathrm{DN}$ (saturation) $-\mathrm{DN}($ sky $))$, where DN(saturation) $=32767$ is the saturation level of the analog-to-digital converter (which is, in turn, set below the full well of the CCD detector) and $n$ is the number of frames summed ( $n=6$ or 7 ). Thus, objects with any saturated pixels in the periphery of the galaxy were rejected, while a very small number of point sources with a few saturated pixels which were seen against a very bright background may not have been rejected.

Excluded windows, whose area was not included in the search, consisted of bad columns, regions around bright stars, regions of large partially resolved foreground galaxies or dwarf galaxies, regions where the nucleus of the Virgo elliptical was saturated, the area covered by the pickoff arm for the guider, etc. Also excluded were the edges of each MASTERG frame where not all the individual frames contribute to this sum because of the spatial shift between the individual exposures. The region that was eliminated due to saturation of the nucleus itself was typically 120 pixels in diameter. The total effective area in $\operatorname{arcmin}^{2}$ of each chip of the MASTERG frame for each of the three galaxies is listed in Table I. Since chips 3 and 4 have more cosmetic defects than do chips 1 or 2 , their effective areas are typically smaller.

Inspection of the frames revealed that there were many obviously extended galaxies. From the final list of real objects, we removed by hand all objects which on a monitor displaying the data at full spatial resolution and at various black/white display levels were obviously extended (i.e., small galaxies). These had typical magnitudes of $g=22-23$ and were few arcsec in size. The number of small galaxies removed per frame is listed in Table I as is the number of larger galaxies which were excluded as windows. The galaxy cluster to the southeast of the nucleus of NGC 4486 noted by Huchra and Brodie (1984) is obvious in the MASTERG frames and in the count rates for small galaxies in Table I. All of the galaxies observed as globular cluster candidates in NGC 4486 by Huchra and Brodie (1984) are obviously galaxies; the smallest of them is at least 6 arcsec in size on the MASTERG frames. Of the globular cluster candidates of Mould, Oke, and Nemec (1986) that were spectroscopically

TABLE I. Characteristics of the detected objects.

\begin{tabular}{ccccc}
\hline \hline Chip & $\begin{array}{c}\text { No. Point Sources } \\
g \leq 25 \mathrm{mag}\end{array}$ & $\begin{array}{c}\text { No. Small } \\
\text { Galaxies }\end{array}$ & $\begin{array}{c}\text { No. Large } \\
\text { Galaxies }\end{array}$ & $\begin{array}{c}\text { Effective Area } \\
\left(\text { arc-min }^{2}\right)\end{array}$ \\
\hline NGC 4406 & & & & \\
1 & 690 & 14 & 3 & 16.14 \\
2 & 661 & 20 & 0 & 16.59 \\
3 & 524 & 18 & 0 & 16.68 \\
4 & 552 & 20 & 0 & 14.52 \\
NGC 4472 & & & & \\
1 & & & & \\
2 & 678 & 14 & 1 & 15.79 \\
3 & 758 & 16 & 1 & 15.56 \\
4 & 869 & 23 & 1 & 15.02 \\
NGC 4486 & 878 & 14 & 3 & \\
1 & & & & 17.87 \\
2 & 1015 & 18 & 0 & 15.55 \\
3 & 1002 & 17 & 4 & 13.92 \\
\hline
\end{tabular}


classified as galaxies or suspected galaxies, most were not rejected as extended, with the exception of numbers 601 , 944, and 969 (in the numbering system of Strom et al. 1981), which are obviously galaxies.

The number of point sources detected is large. Table I lists the total number of point sources whose photometry indicated that they are brighter than $g=25.0$ mag detected in the MASTERG frames. When there are one thousand detected sources per frame, the average separation of objects is about 25 pixels. It should also be noted that, except for the removal of galaxies, all manipulations ran without manual intervention, so that whatever biases may exist are identical in all the frames. The only other manual procedure was the selection of a minimum value for the point-source detection code, and unless one is pushing fainter than the limiting magnitude discussed here, that is irrelevant.

\section{b) Cluster Photometry}

An aperture-photometry code was used for each point source, where the sky was determined from an annulus of radius $12-15$ pixels from the source center, and the magnitude within an aperture 5 pixels in radius. This code had its own precise centering routine. Sources were accepted as real whose centers were not shifted by more than 1.5 pixels in either dimension between the point-source detection code and the aperture-centering code. Furthermore, the magnitude determined from the aperture code had to be within 0.8 mag of that determined from a $7 \times 7$ pixel box sum around the source carried out as part of the point-source detection code, when the sky determination of the aperture code was used. Examination of the source lists showed that in the outer parts of the galaxies nearly all sources were real, and all real sources were found. The innermost parts where the residual background was relatively large and variable had more problems, and a separate procedure was used for the region closest to the nucleus. A subset of each chip of the MASTERG frame which included the area closest to the nucleus of the galaxy was median filtered by a $25 \times 25$ pixel box, and then the point-source detection code was run on the median-filter-subtracted frame. The cutoff for these frames was set rather higher, since otherwise many spurious sources were found. The $g$ magnitudes were then measured on the median-filtered frames, and a small correction factor (typically several hundredths of a magnitude) was applied. The correction factor was determined by comparing magnitudes of objects on the original frames and on the median-filtersubtracted frames. The subsets for which this was done are subsequently referred to as the nuclear subsets.

Aperture corrections to large apertures from the 3.3- arcsec-diameter aperture used in the photometry code were determined from isolated bright stars in each frame. There were, on average, only six suitable relatively bright uncrowded stars per $800 \times 800$ pixel frame; the aperture corrections for the MASTERG frames of NGC 4406, 4472, and 4486 ranged from 0.08 to $0.10 \mathrm{mag}$.

The $r$ and $i$ magnitudes were determined from their respective frames using the master source lists from the $g$ frames. The only subtlety is that the image scale is slightly different due to the presence of several glass elements in transmission within the optical path. Thus, the spatial shift between the MASTERG and $r$ or $i$ frames was determined at each corner and at the center of each of the four frames composing the total 4-Shooter image. The appropriate shift for each subset was used, depending on where it was located within the frame. Aperture corrections for the $r$ frames were $0.07 \pm 0.01 \mathrm{mag}$ and typical images had a FWHM of 1.1 arcsec. (Notice that the small difference of not more than 0.03 mag between the aperture corrections for the MASTERG and the $r$ frames of each galaxy implies that the shifting and summing to create the MASTERG frames did not significantly degrade the point-source image profile.) However, the seeing during the $i$ exposures was somewhat worse, ranging from 1.4 arcsec for NGC 4486 to 2.0 arcsec for NGC 4472. Hence the aperture corrections ranged from $0.17 \mathrm{mag}$ (for NGC 4486) to $0.40 \mathrm{mag}$ (for NGC 4472). Even in the nuclear regions, we did not median filter the $r$ or $i$ frames.

The photometric zero points and the transformations from instrumental to standard magnitudes and colors used for the clusters are identical to those used in Paper I for the light of the underlying galaxy. The transformation between the $g, r, i$ system and the Johnson $B, V$ system adopted here is that of Cohen (1985). For a $g-r$ color of 0.40 mag, typical of the median of the globular cluster systems discussed here (see Sec. V), $V=g-0.15 \mathrm{mag}$, and $B=g+0.65 \mathrm{mag}$. In practice, an object whose $g$ magnitude is 25.0 had a total of $\approx 5000 \mathrm{DN}$ (equivalent to $\approx 10000$ electrons) above the sky within the 5 pixel aperture on the MASTERG frames. Its brightest pixel had a net signal above the sky of $\approx 400 \mathrm{DN}$ or a $4.7 \sigma$ detection above the background. For $r$, the total net signal above the sky of a source whose magnitude was 23.0 was $\approx 400 \mathrm{DN}$. Of course, one must recall that the sky level on the MASTERG frames was $\approx 17000 \mathrm{DN} /$ pixel, and a substantial spatially varying contribution from the underlying galaxy halo light was also present. The actual limiting magnitude and magnitude histograms are discussed in Sec. IV.

The location of each object was determined both in radius with respect to the center of the galaxy and in semimajor axis using the ellipse fits of Paper I.

Tables II and III list the point sources detected in the fields of NGC 4406 and NGC 4472 with $g \leqslant 22.1$ mag. Table II contains 164 sources, while Table III has 292 entries. No such table for NGC 4486 is included, as its brighter clusters are listed in the previous study by Strom et al. (1981). The positions are given in arcsec in a coordinate system centered on the nucleus of each galaxy. Positive $x$ and $y$ correspond to west and north, respectively.

\section{DEFINING THE BACKGROUND}

No additional frames were available to determine the background field that reached as deep as the galaxy frames themselves. We used, therefore, the parts of the MASTERG frames of NGC 4406 and NGC 4472 that were furthest (in semimajor axis) from the nucleus of the galaxy to define the background field. This is clearly acceptable for NGC 4406, since there are few clusters, and the background is reached quickly. It is somewhat less so for NGC 4472, since the cluster system is more extensive. Note that the three galaxies are within $5^{\circ}$ of each other on the sky and have a total range in galactic latitude from $70.2^{\circ}$ (NGC 4472 ) to $74.6^{\circ}$ (NGC 4406). The total area used as the background field is 9.2 $\operatorname{arcmin}^{2}$ in NGC 4406 and $3.1 \operatorname{arcmin}^{2}$ in NGC 4472, and includes a sample of 407 sources brighter than $g=25.0$. The magnitude histogram $\sigma$ for the point sources detected in the background field (number/arcmin $2 / 0.1 \mathrm{mag}$ bin) is plotted in Fig. 1, together with the errors for each point based on Poisson statistics. The solid line in the figure is a fit of this histogram: 


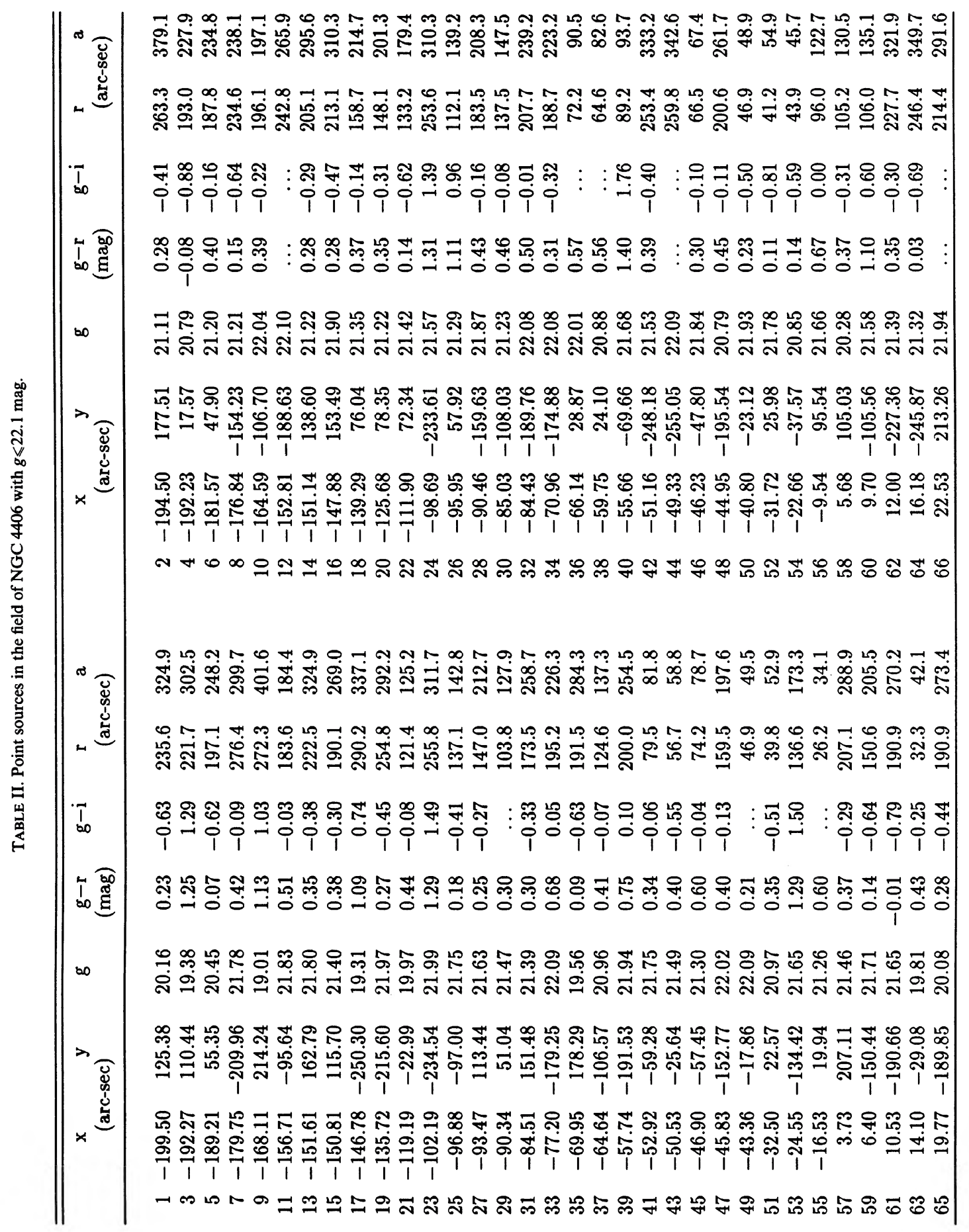




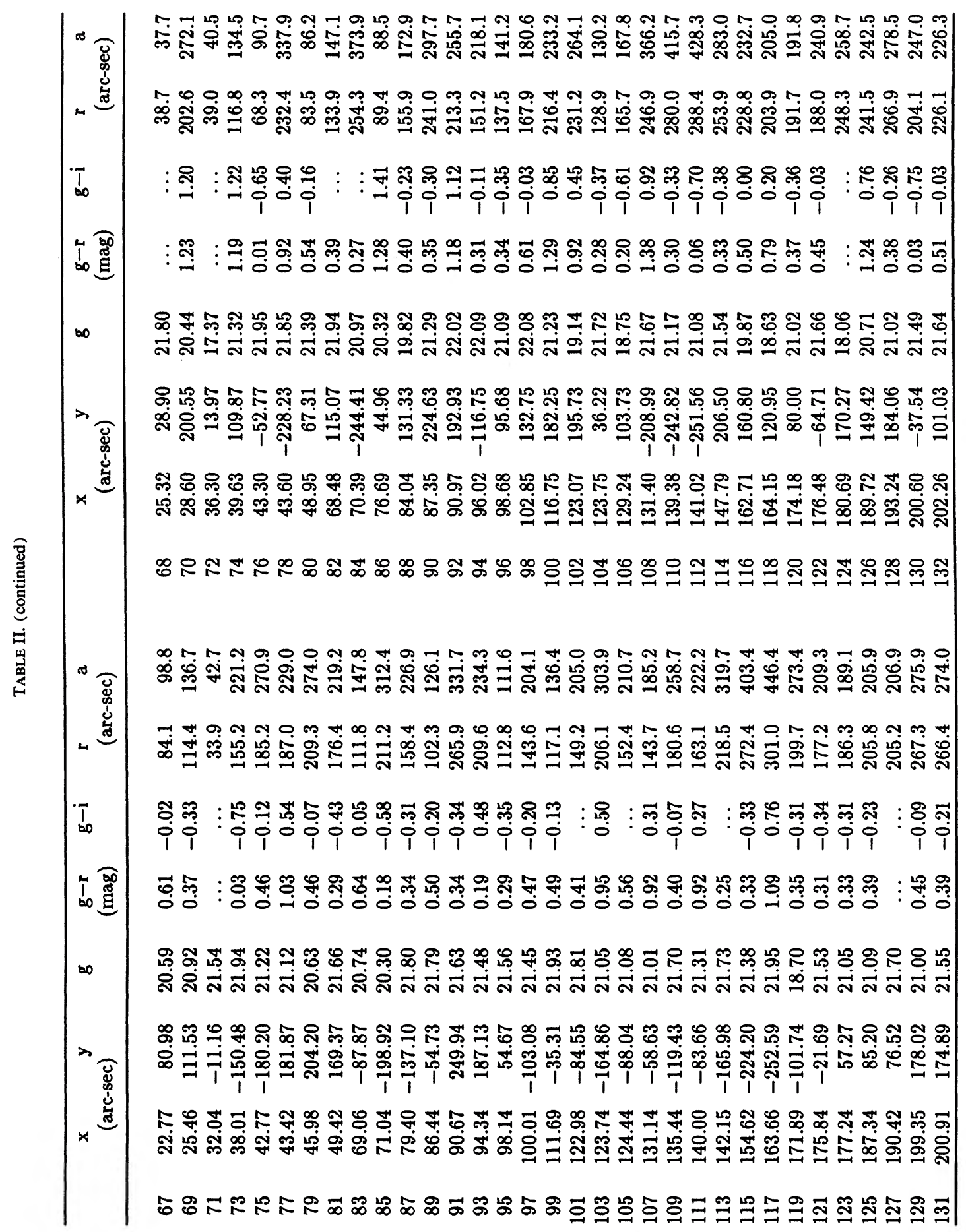




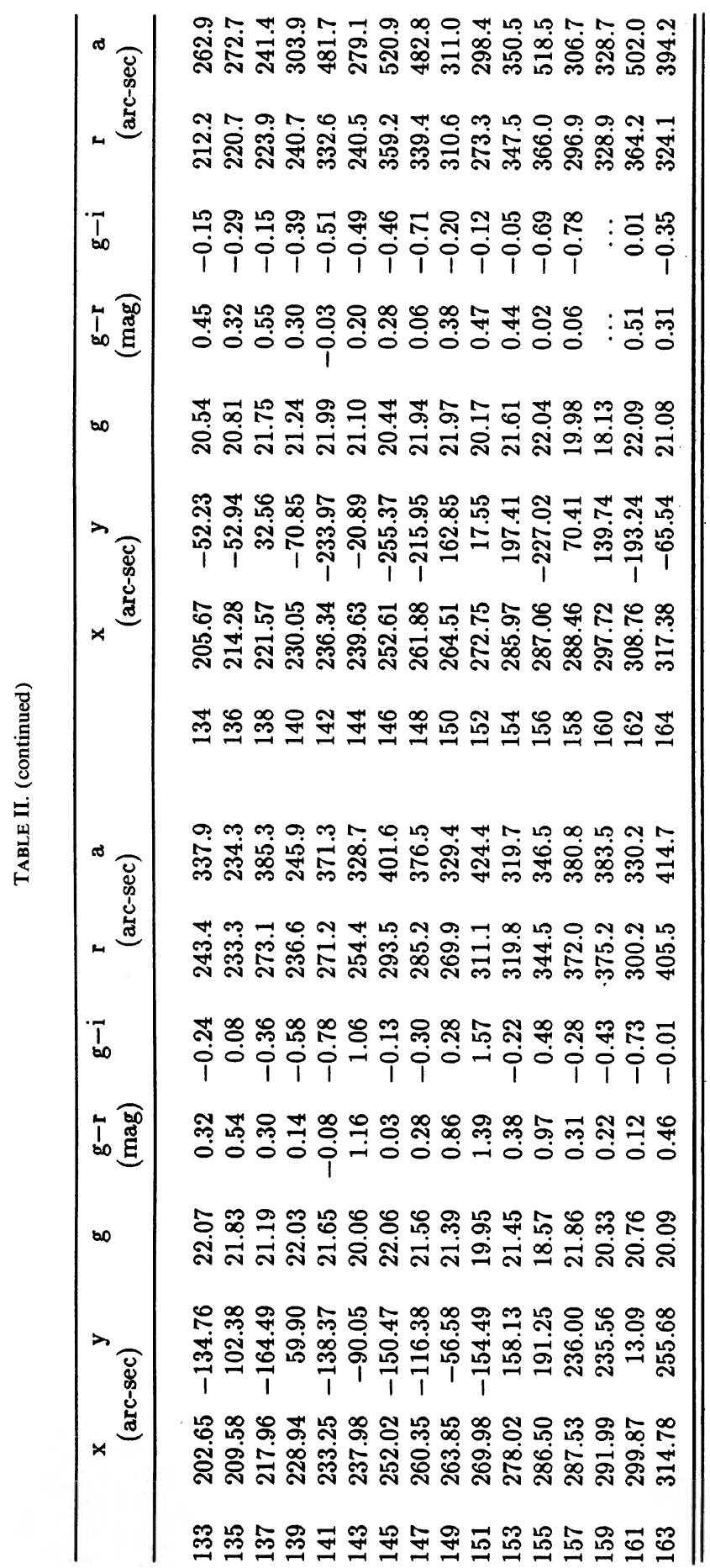




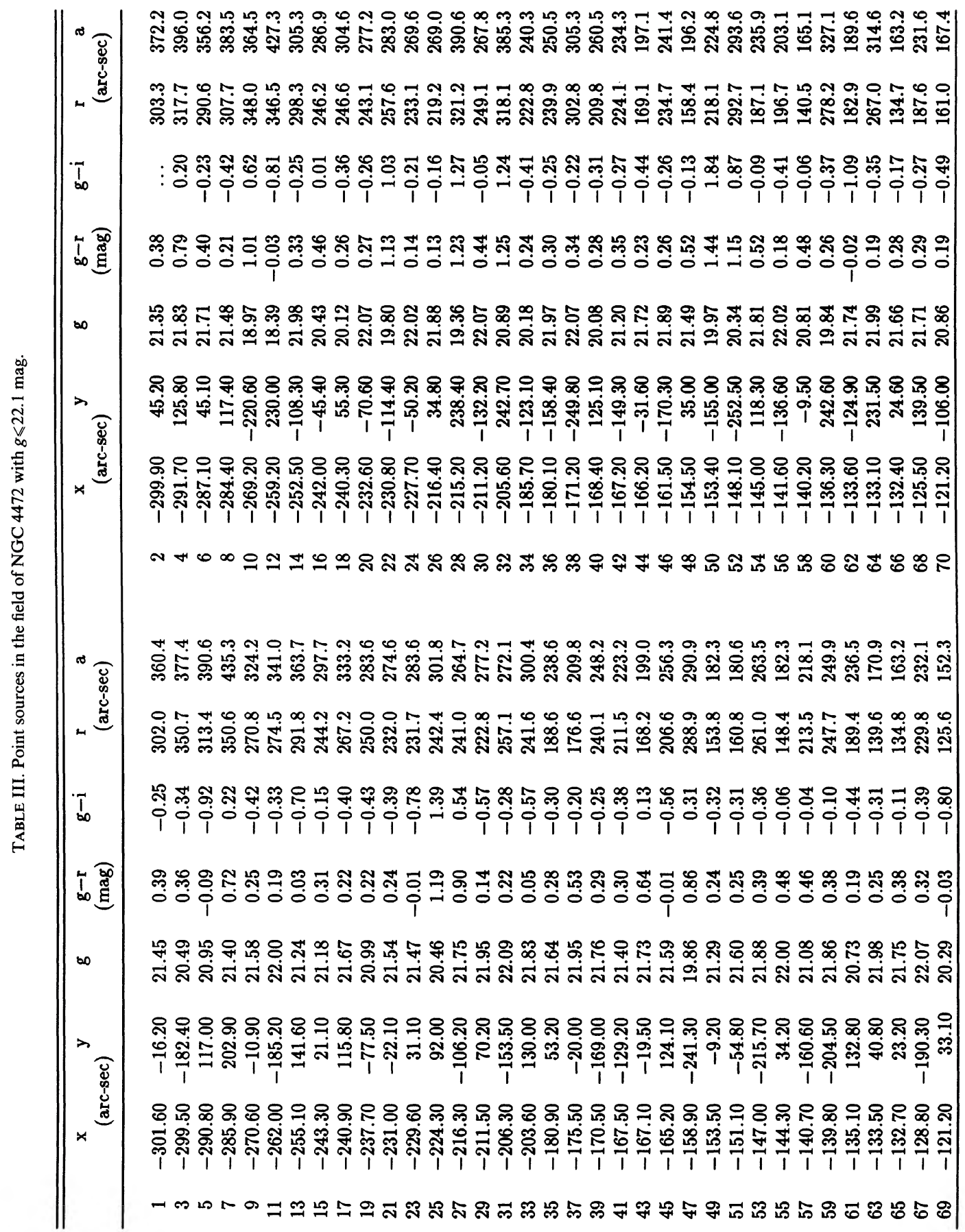




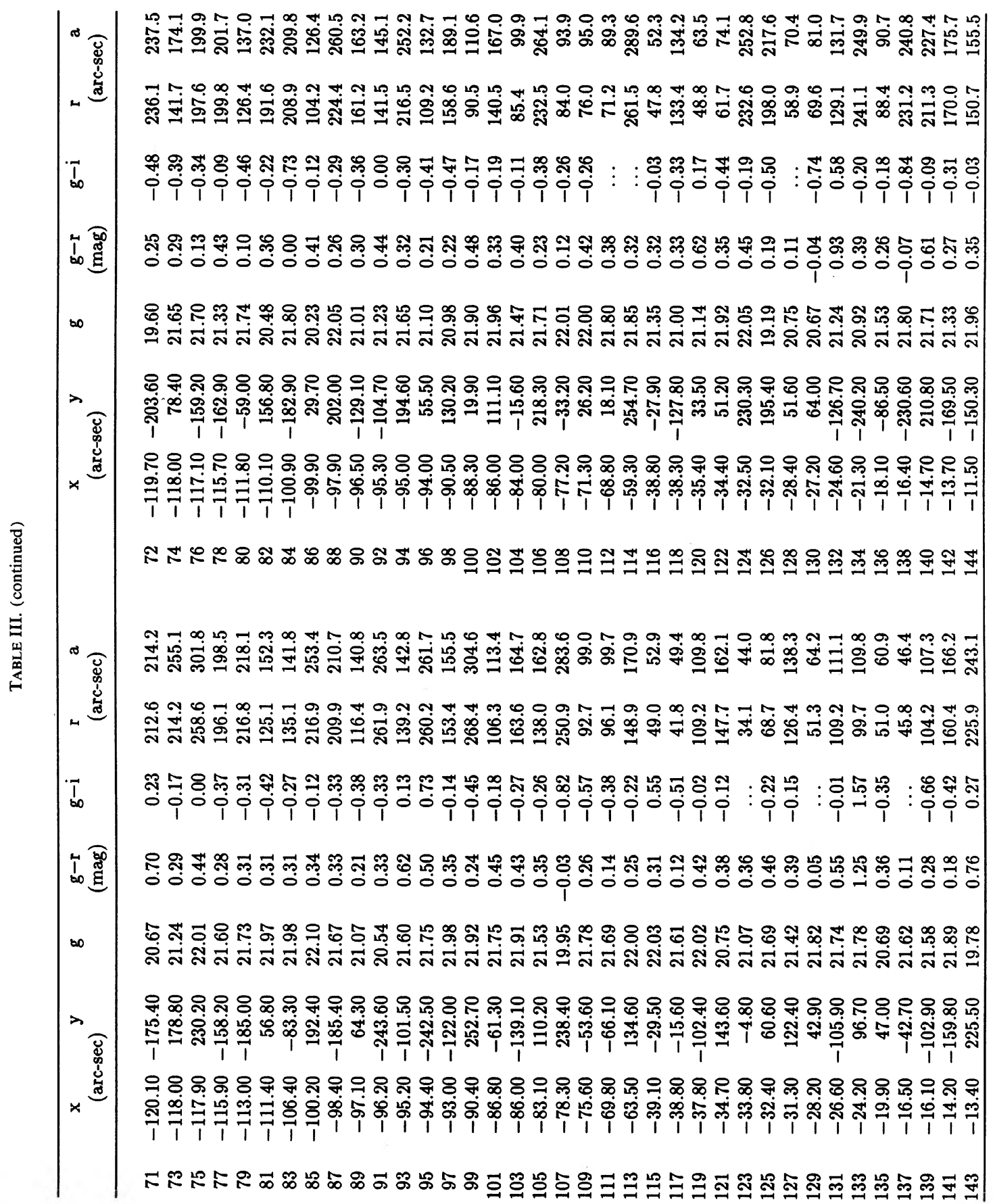




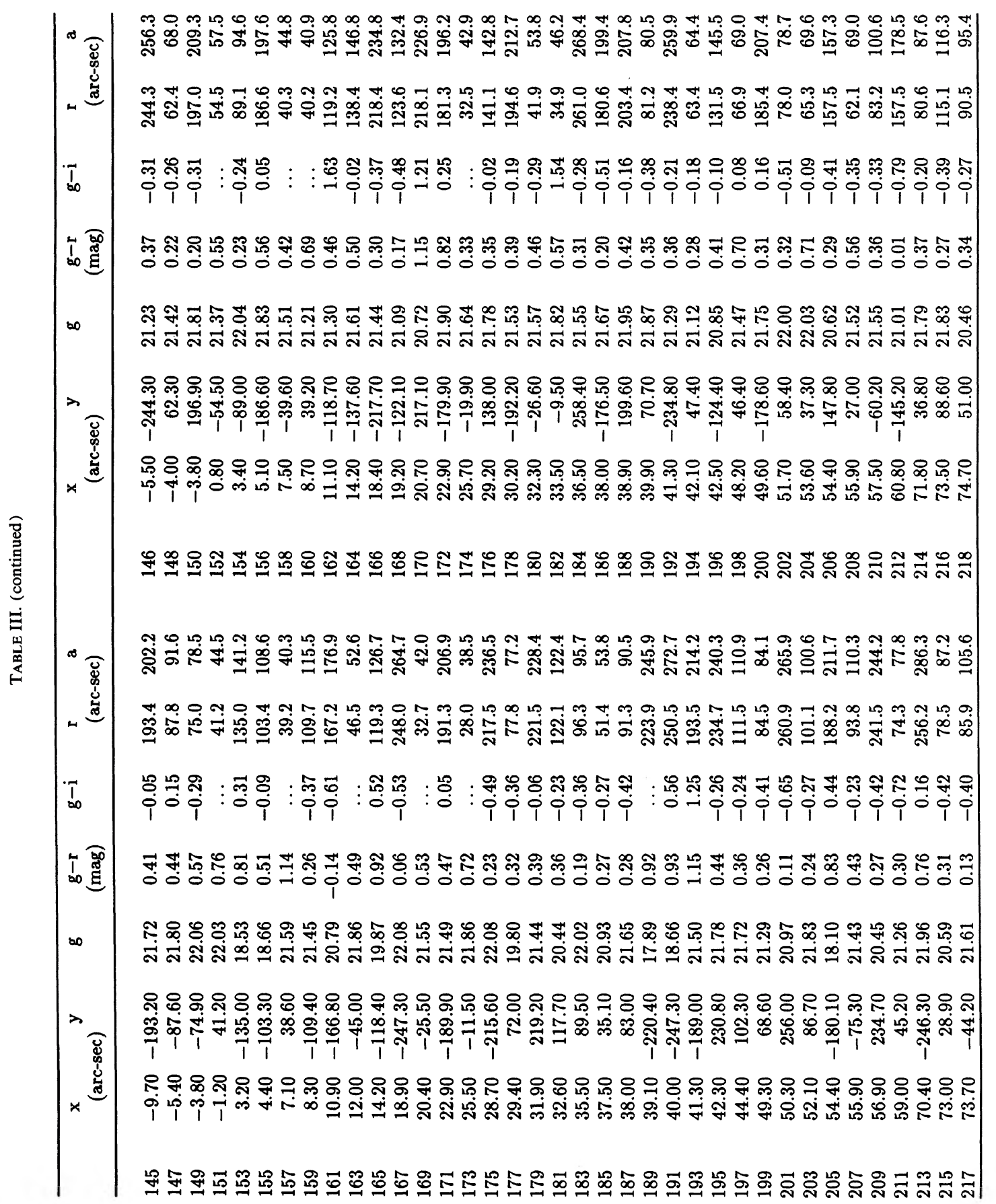




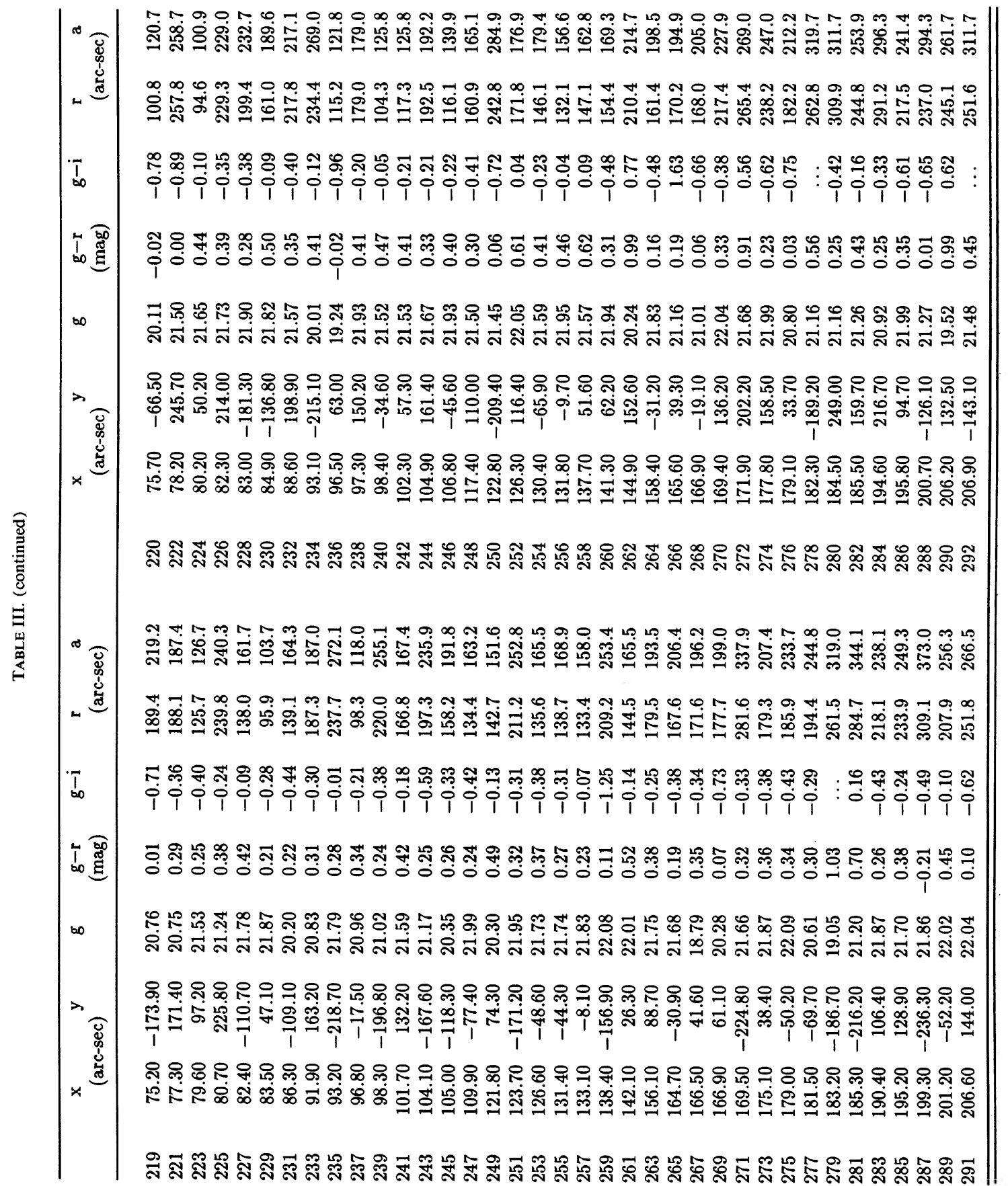




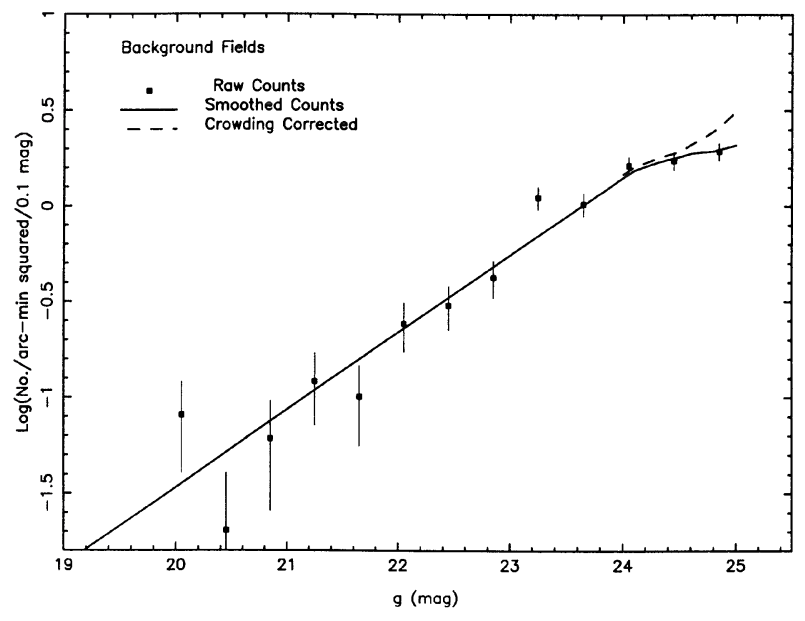

FIG. 1. The count rate of point sources in the background fields (number/ $\operatorname{arcmin}^{2} / 0.1 \mathrm{mag}$ ) is shown as a function of $g$ magnitude. The squares represent the actual counts, while the solid line is a smoothed fit used in subsequent analysis. The dashed line is the smoothed background with corrections for incompleteness caused by crowding.

$$
\log (\sigma)=-9.548+0.404 \times g .
$$

This relationship was used as the actual background magnitude distribution for $g \leqslant 24.1 \mathrm{mag}$. For $g>24.1 \mathrm{mag}$, the background counts were fit by the smooth curve indicated in Fig. 1. The dashed line shows the faintest magnitude with the crowding corrections to the source counts described in Sec. IV $b$.

The slope of the background counts $\Delta \log (\sigma) / 1.0 \mathrm{mag}$ of $0.40 \pm 0.03$ dex compares well with that of Tyson and Jarvis (1979; see also Jarvis and Tyson 1981; Kron 1980; and Harris and Smith 1976, 1981), but is somewhat shallower than that of van den Bergh, Pritchet, and Grillmair (1985). The background we adopt agrees with that adopted by van den Bergh, Pritchet, and Grillmair at $g \approx 23.1 \mathrm{mag}(B \approx 23.8$ mag).

\section{THE MAGNITUDE DISTRIBUTION OF THE CLUSTERS}

\section{a) The Limiting Magnitude}

The number of detected electrons for a point source in a MASTERG frame is a poor indicator of the reliability of the detection. The true limiting magnitude is set by fluctuations in the large subtracted background and by local irregularities in the CCD detectors that are not properly removed by the simple flatfielding procedures adopted here. A careful comparison of the detected source lists with the actual frames shows that $g=25.0$ is the limit to which the detected source lists are reliable. Fainter than that level, especially in the parts of the frame that are fairly close to the nucleus of the galaxy, many spurious point sources are interspersed among the true objects. The magnitude histograms for the globular cluster systems were thus limited to $g \leqslant 25 \mathrm{mag}$. To further ensure that no spurious sources were accepted, the point sources found by the computer code were individually checked for reliability between $24.5 \leqslant g \leqslant 25.0 \mathrm{mag}$, and a few spurious ones were eliminated. One should note that no other part of the present work utilizes clusters fainter than $g=24.0$ mag; for the brighter clusters there is no question that all detections are real and that (essentially) all real objects in the area searched have been detected.

\section{b) Completeness of the Detections}

The completeness of the detection of globular clusters is a function of both magnitude and crowding (i.e., distance from the nucleus of the galaxy). To derive the completeness corrections, a code was written which added point sources with a spatial profile identical to that of the real bright point sources in the MASTERG frames. These point sources were added in a rectangular grid 50 pixels apart with small random offsets of the entire grid in $x$ and $y$ for each test run. Points that fell in the area of excluded windows were subsequently ignored. The light intensity in each pixel of the added point sources was scaled to the brightness level that was being tested for completeness, which ranged from 23.0 to $25.0 \mathrm{mag}$ in steps of $0.5 \mathrm{mag}$. Recovery was then attempted through the regular codes used to detect the globular clusters, and the rate of recovery used to define the completeness corrections. The nuclear sections are quite crowded and the spatial variation of the residual background is large due to the large subtracted light of the galaxy. Therefore, no recovery was attempted for $R \leqslant 50$ arcsec, where $R$ is the radial distance from the center of the galaxy. Typical trials involved adding 70-90 point sources to the inner area and 600670 point sources to the outer area. The fraction of point sources that were not recovered is given in Table IV for the inner and outer regions of NGC 4406 and for NGC 4486 as a function of $g$ magnitude, where for present purposes "inner" refers to 50-70 arcsec, and "outer" refers to $R>70$ arcsec. We see that the crowding, which is obviously worse in NGC 4486 than in NGC 4406, and is also worse in the inner section of each galaxy than in the outer section, is not of serious concern until $g>24$ mag.

We assume that the crowding corrections for NGC 4472 are intermediate between those determined for NGC 4486 and NGC 4406.

An indication of the accuracy of the crowding corrections can be obtained by examining the faint end of the magnitude histogram of the background sources. We compare the crowding-corrected counts shown by the dashed line in Fig. 1 with the extrapolation of the power law fit to the brighter background sources. Over the interval $24<g<25$ mag, there is a maximum discrepancy between the crowding corrected counts $\sigma(g)$ and the extrapolated line of $16 \%$ of the value of $\sigma(g)$.

\section{c) The Magnitude Histograms}

The histograms of the number of detected point sources as a function of $g$ magnitude were derived separately for the nuclear region, the inner area, and the outer area in each galaxy. (The division was based only on the value of the

TABLE IV. Recovery rate of added point sources.

\begin{tabular}{cccc}
\hline \hline Galaxy & $\begin{array}{c}\mathrm{g} \\
\text { (mag) }\end{array}$ & $\begin{array}{c}\text { Inner Section } \\
\text { (\% Not Recovered) }\end{array}$ \\
\hline NGC. 4406 & 23.0 & 4 & Outer Section \\
& 23.5 & 0 & 2 \\
& 24.0 & 8 & 4 \\
& 24.5 & 14 & 6 \\
NGC 4.186 & 25.0 & 41 & 33 \\
& 23.0 & 13 & 6 \\
& 23.5 & 13 & 6 \\
& 24.0 & 21 & 11 \\
& 24.5 & 35 & 16 \\
& 25.0 & 55 & 39 \\
\hline \hline
\end{tabular}


semimajor axis for each object with respect to the nucleus of the galaxy). We then used the total area within each of the three groups (minus all windows and the guider arm if present) combined with the background derived as described in Sec. III. The resulting histograms are tabulated in Tables $\mathrm{V}(\mathrm{a})-\mathrm{V}(\mathrm{c})$, where $N$ is the number of detected point sources/arcmin ${ }^{2} / 0.2 \mathrm{mag}$ bin, and $N_{\mathrm{c}}$ is derived from $N$ by correcting for the background sources as well as the completeness of both the field and background counts. Because no crowding corrections were evaluated for the nuclear region, the magnitude histograms for that area are cut off at a relatively bright limit. The data, together with the uncertainties, are plotted for the nuclear, inner, and outer sections of NGC 4472 and NGC 4486 in Figs. 3(a)-3(c) and 4(a)4 (c), while Fig. 2 shows only the inner region of NGC 4406. The uncertainties are based only on the Poisson statistics of the number of detected objects both in the smoothed background and in the actual counts; they do not include uncertainties in the crowding corrections.

While discussing the magnitude histograms derived from the present date, one should also bear in mind the uncertainties in the $g$ magnitudes themselves. The external errors (i.e., the transformations to a standard photometric system involving the observations of standard stars) are insignificant compared to the internal errors in the magnitudes. The same

TABLE V(a). Number-magnitude histogram for point sources in the field of NGC 4406.

\begin{tabular}{|c|c|c|c|c|}
\hline \multirow{3}{*}{$\frac{\begin{array}{c}\bar{g} \\
(\mathrm{mag})\end{array}}{19.8}$} & \multicolumn{2}{|c|}{ Nuclear Area } & \multicolumn{2}{|c|}{ Inner Area } \\
\hline & \multicolumn{3}{|c|}{ (No. $/ \operatorname{arc}-\min ^{2} / 0.2 \mathrm{mag}$ ) } & $\mathrm{N}_{c}$ \\
\hline & $0.66^{1}$ & 0.62 & $0.00^{2}$ & -0.05 \\
\hline 20.0 & 0.00 & -0.06 & 0.15 & 0.10 \\
\hline 20.2 & 0.00 & -0.08 & 0.00 & -0.08 \\
\hline 20.4 & 0.00 & -0.09 & 0.15 & 0.07 \\
\hline 20.6 & 0.00 & -0.12 & 0.15 & 0.04 \\
\hline 20.8 & 0.66 & 0.54 & 0.15 & 0.02 \\
\hline 21.0 & 0.66 & 0.52 & 0.00 & -0.17 \\
\hline 21.2 & 0.66 & 0.48 & 0.15 & -0.06 \\
\hline 21.4 & 0.66 & 0.45 & 0.29 & 0.08 \\
\hline 21.6 & 0.66 & 0.41 & 0.44 & 0.19 \\
\hline 21.8 & 1.32 & 1.01 & 0.44 & 0.14 \\
\hline 22.0 & 1.32 & 0.95 & 0.29 & -0.15 \\
\hline 22.2 & 1.98 & 1.53 & 1.16 & 0.74 \\
\hline 22.4 & 0.66 & 0.12 & 2.33 & 1.84 \\
\hline 22.6 & 1.98 & 1.26 & 1.02 & 0.33 \\
\hline 22.8 & 1.98 & 1.09 & 1.16 & 0.31 \\
\hline 23.0 & 3.95 & 2.86 & 1.45 & 0.41 \\
\hline 23.2 & 2.64 & 1.28 & 2.47 & 1.23 \\
\hline 23.4 & 7.25 & 5.58 & 1.31 & -0.23 \\
\hline 23.6 & $\ldots$ & $\ldots$ & 2.62 & 0.79 \\
\hline 23.8 & $\ldots$ & $\ldots$ & 4.65 & 2.61 \\
\hline 24.0 & $\ldots$ & $\ldots$ & 4.51 & 2.12 \\
\hline 24.2 & $\ldots$ & $\ldots$ & 6.40 & 3.87 \\
\hline 24.4 & $\ldots$ & $\ldots$ & 4.65 & 1.90 \\
\hline 24.6 & $\ldots$ & $\ldots$ & 3.63 & 0.61 \\
\hline 24.8 & $\ldots$ & $\ldots$ & 2.18 & -2.74 \\
\hline
\end{tabular}

1 The Nuclear Area has $25 \leq \mathrm{A} \leq 60$ arc-sec and includes a total area searched of 1.52 arc$\min ^{2}$.

2 The Inner Area has $60<\mathrm{A}<130$ arc-sec and includes a total area searched of 6.88 arc$\min ^{2}$.
TABLE V (b). Number-magnitude histogram for point sources in the field of NGC 4472

\begin{tabular}{ccccccr}
\hline \hline & \multicolumn{2}{c}{ Nuclear Area } & \multicolumn{2}{c}{ Inner Area } & \multicolumn{2}{c}{ Outer Area } \\
$\bar{g}$ & $\mathrm{~N}$ & $\mathrm{~N}_{\mathrm{c}}$ & $\mathrm{N}$ & $\mathrm{N}_{\mathrm{c}}$ & $\mathrm{N}$ & $\mathrm{N}_{c}$ \\
& \multicolumn{2}{c}{ (Number/arc-min $\left.{ }^{2} / 0.2 \mathrm{mag}\right)$} \\
\hline 19.8 & $0.00^{1}$ & -0.05 & $0.26^{2}$ & 0.20 & $0.08^{3}$ & 0.02 \\
20.0 & 0.00 & -0.06 & 0.00 & -0.06 & 0.08 & 0.01 \\
20.2 & 0.00 & -0.08 & 0.26 & 0.18 & 0.13 & 0.06 \\
20.4 & 0.00 & -0.09 & 0.26 & 0.16 & 0.13 & 0.04 \\
20.6 & 0.00 & -0.12 & 0.38 & 0.27 & 0.08 & -0.04 \\
20.8 & 0.00 & -0.14 & 0.13 & -0.01 & 0.23 & 0.09 \\
21.0 & 1.24 & 1.07 & 0.13 & -0.03 & 0.27 & 0.10 \\
21.2 & 0.62 & 0.42 & 0.64 & 0.44 & 0.34 & 0.14 \\
21.4 & 1.24 & 1.00 & 0.64 & 0.41 & 0.30 & 0.07 \\
21.6 & 4.32 & 4.04 & 1.53 & 1.27 & 0.51 & 0.23 \\
21.8 & 1.85 & 1.51 & 1.66 & 1.36 & 0.87 & 0.54 \\
22.0 & 1.24 & 0.82 & 1.28 & 0.90 & 0.87 & 0.47 \\
22.2 & 3.71 & 3.20 & 2.81 & 2.43 & 1.14 & 0.66 \\
22.4 & 3.09 & 2.48 & 2.68 & 2.23 & 1.22 & 0.64 \\
22.6 & 0.62 & -0.15 & 1.91 & 1.32 & 1.25 & 0.56 \\
22.8 & 6.18 & 5.30 & 3.06 & 2.45 & 1.63 & 0.81 \\
23.0 & 6.80 & 5.74 & 2.68 & 1.89 & 2.36 & 1.39 \\
23.2 & 6.80 & 5.52 & 4.47 & 3.70 & 2.41 & 1.24 \\
23.4 & 8.65 & 7.10 & 3.96 & 2.92 & 2.72 & 1.32 \\
23.6 & $\ldots$ & $\ldots$ & 4.98 & 3.82 & 2.89 & 1.21 \\
23.8 & $\ldots$ & $\ldots$ & 6.89 & 5.71 & 4.35 & 2.43 \\
24.0 & $\ldots$ & $\ldots$ & 6.38 & 4.68 & 4.12 & 1.70 \\
24.2 & $\ldots$ & $\ldots$ & 7.40 & 5.55 & 4.24 & 1.36 \\
24.4 & $\ldots$ & $\ldots$ & 6.25 & 4.39 & 4.39 & 1.47 \\
24.6 & $\ldots$ & $\ldots$ & 3.70 & 1.10 & 3.80 & 0.66 \\
24.8 & $\ldots$ & $\ldots$ & 3.57 & 0.67 & 4.18 & 0.78 \\
\hline \hline
\end{tabular}

1. The nuclear area has $25 \leq \mathrm{A} \leq 60$ arc-sec and includes a total area searched of 1.62 arc- $\min ^{2}$.

2. The inner area has $60<\mathrm{A}<130$ arc-sec and includes a total area searched of 7.84 arc-min ${ }^{2}$.

3. The outer area has $A \geq 130$ arc-sec and includes a total area searched of 52.64 arc- $\min ^{2}$.

statistical tests described in Sec. IV $b$ that were used to derive the completeness corrections as a function of magnitude can be utilized here. These involve adding to the MASTERG frames additional spurious point sources and then recovering them through the same codes used to analyze the true point-source detections. The standard deviation of the difference between the magnitude of the added point source and that recovered from the codes operating on the summed MASTERG frames is less than $0.2 \mathrm{mag}$ for $g \leqslant 23.5 \mathrm{mag}$. At $g=24.5 \mathrm{mag}, \sigma(g)$ reaches $0.4 \mathrm{mag}$.

We first examine the slope of the $N_{c}$-magnitude distribution. It is marginally shallower in NGC 4486 than in NGC 4472. This is unlikely to be a result of relative errors in the crowding corrections, since the same relative difference is seen when comparing the inner and the outer areas of both galaxies. The NGC 4486 magnitude distribution is best fit by a Gaussian with $\sigma=1.5 \mathrm{mag}$, while that of NGC 4472 has $\sigma \approx 1.2 \mathrm{mag}$. The statistical significance of the difference in the $N_{\mathrm{c}}$-magnitude distribution between NGC 4472 and NGC 4486 is hard to assess. While a Kolmogorov-Smirnov test on the raw source detections yields a very high probability that the two distributions are different, this is only to be expected, since each distribution is the sum of a fixed background and a contribution of globular clusters with a very different luminosity function. The difference in richness of 
TABLE V (c). Number-magnitude histogram for point sources in the field of NGC 4486.

\begin{tabular}{|c|c|c|c|c|c|c|}
\hline \multirow[b]{2}{*}{$\bar{g}$} & \multicolumn{2}{|c|}{ Nuclear Area } & \multicolumn{2}{|c|}{ Inner Area } & \multicolumn{2}{|c|}{ Outer Area } \\
\hline & \multicolumn{4}{|c|}{ (Number $/ \operatorname{arc}-\mathrm{min}^{2} / 0.2 \mathrm{mag}$ ) } & $\mathbf{N}$ & $\mathrm{N}_{c}$ \\
\hline 19.8 & $0.73^{1}$ & 0.68 & $0.23^{2}$ & 0.18 & $0.04^{3}$ & -0.00 \\
\hline 20.0 & 0.00 & -0.06 & 0.35 & 0.29 & 0.11 & 0.05 \\
\hline 20.2 & 0.73 & 0.65 & 0.24 & 0.16 & 0.07 & -0.01 \\
\hline 20.4 & 0.73 & 0.64 & 0.35 & 0.26 & 0.22 & 0.13 \\
\hline 20.6 & 0.73 & 0.62 & 0.71 & 0.59 & 0.41 & 0.29 \\
\hline 20.8 & 0.73 & 0.60 & 0.47 & 0.33 & 0.50 & 0.36 \\
\hline 21.0 & 1.46 & 1.30 & 2.00 & 1.83 & 0.52 & 0.35 \\
\hline 21.2 & 4.39 & 4.19 & 1.18 & 0.98 & 0.78 & 0.58 \\
\hline 21.4 & 5.12 & 4.89 & 2.58 & 2.38 & 0.70 & 0.47 \\
\hline 21.6 & 4.39 & 4.10 & 3.29 & 3.08 & 0.96 & 0.69 \\
\hline 21.8 & 6.59 & 6.24 & 2.94 & 2.69 & 1.24 & 0.92 \\
\hline 22.0 & 10.98 & 10.56 & 3.88 & 3.63 & 1.07 & 0.68 \\
\hline 22.2 & 5.12 & 4.62 & 3.99 & 3.72 & 1.33 & 0.87 \\
\hline 22.4 & 8.05 & 7.45 & 4.93 & 4.70 & 1.93 & 1.40 \\
\hline 22.6 & 10.98 & 10.25 & 5.05 & 4.80 & 2.07 & 1.44 \\
\hline 22.8 & $\ldots$ & $\ldots$ & 5.05 & 4.74 & 2.06 & 1.28 \\
\hline 23.0 & $\ldots$ & $\cdots$ & 6.34 & 6.13 & 2.28 & 1.35 \\
\hline 23.2 & $\ldots$ & $\ldots$ & 6.81 & 6.59 & 3.11 & 2.04 \\
\hline 23.4 & $\ldots$ & $\ldots$ & 7.87 & 7.72 & 3.04 & 1.74 \\
\hline 23.6 & $\ldots$ & $\ldots$ & 8.81 & 8.71 & 3.26 & 1.70 \\
\hline 23.8 & $\ldots$ & $\ldots$ & 7.05 & 6.39 & 3.61 & 1.75 \\
\hline 24.0 & $\ldots$ & $\ldots$ & 8.81 & 8.23 & 3.98 & 1.68 \\
\hline 24.2 & $\ldots$ & $\ldots$ & 6.46 & 5.05 & 4.02 & 1.27 \\
\hline 24.4 & $\ldots$ & $\ldots$ & 4.93 & 3.34 & 4.30 & 1.59 \\
\hline 24.6 & $\ldots$ & $\ldots$ & 4.35 & 2.78 & 4.26 & 1.53 \\
\hline 24.8 & $\ldots$ & $\ldots$ & 2.93 & 0.42 & 3.74 & 0.55 \\
\hline
\end{tabular}

1. The nuclear area has $32 \leq \mathrm{A} \leq 60$ arc-sec and includes a total area searched of $1.37 \mathrm{arc}-\mathrm{min}^{2}$.

2. The inner area has $60<\mathrm{A}<130$ arc-sec and includes a total area searched of 8.51 arc-min ${ }^{2}$.

3. The outer area has $A \geq 130$ arc-sec and includes a total area searched of $54.01 \mathrm{arc}-\mathrm{min}^{2}$.

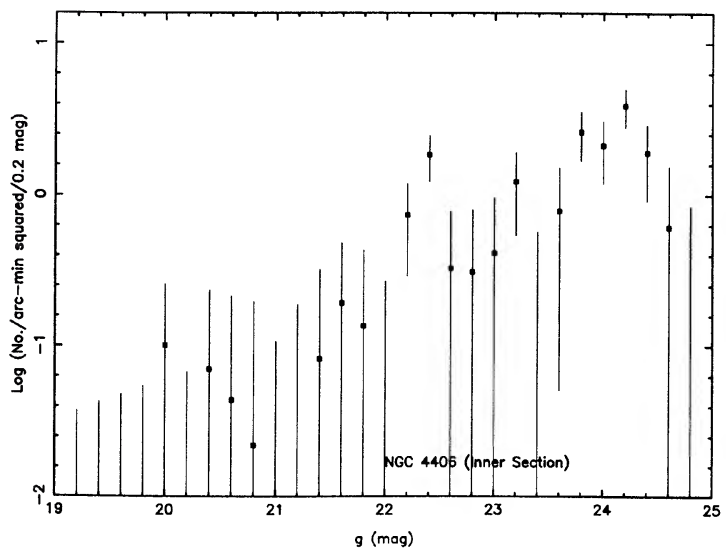

Fig. 2. The net counts above the background per $\operatorname{arcmin}^{2}$ in $0.2 \mathrm{mag}$ bins for point sources in the inner region of NGC 4406 are plotted. Completeness corrections have been applied. The Poisson error bars are shown for each point. the globular cluster system between the two galaxies alone will produce such a result. On the other hand, once one subtracts the background and bins the data, a $\chi^{2}$ test is more appropriate. With the small number of bins, the $\chi^{2}$ test suggests that the difference in the $N_{c}$-magnitude distributions between NGC 4472 and NGC 4486 is not statistically significant.

The peak in the $N_{\mathrm{c}}$-magnitude distribution appears to have been reached in the present data, although only barely before the crowding corrections become so large that the count rates are meaningless. In spite of our efforts to correct for incompleteness, the turnover in the luminosity function of the globular clusters in NGC 4486 becomes fainter in the less crowded outer area of the galaxy, suggesting that the
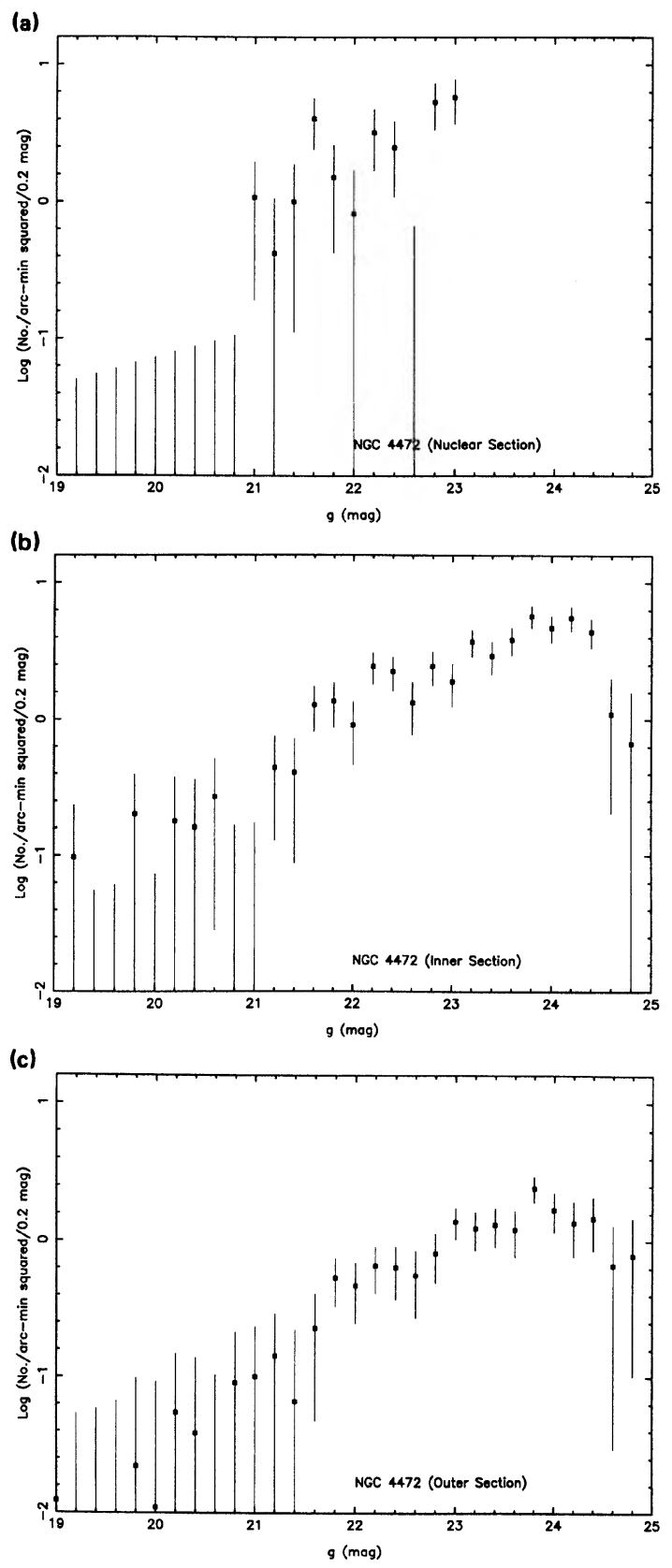

Fig. 3. Same as Fig. 2 for the nuclear section of NGC 4472 (Fig. 3(a)), the inner section (Fig. 3(b)), and the outer section (Fig. 3(c)). 

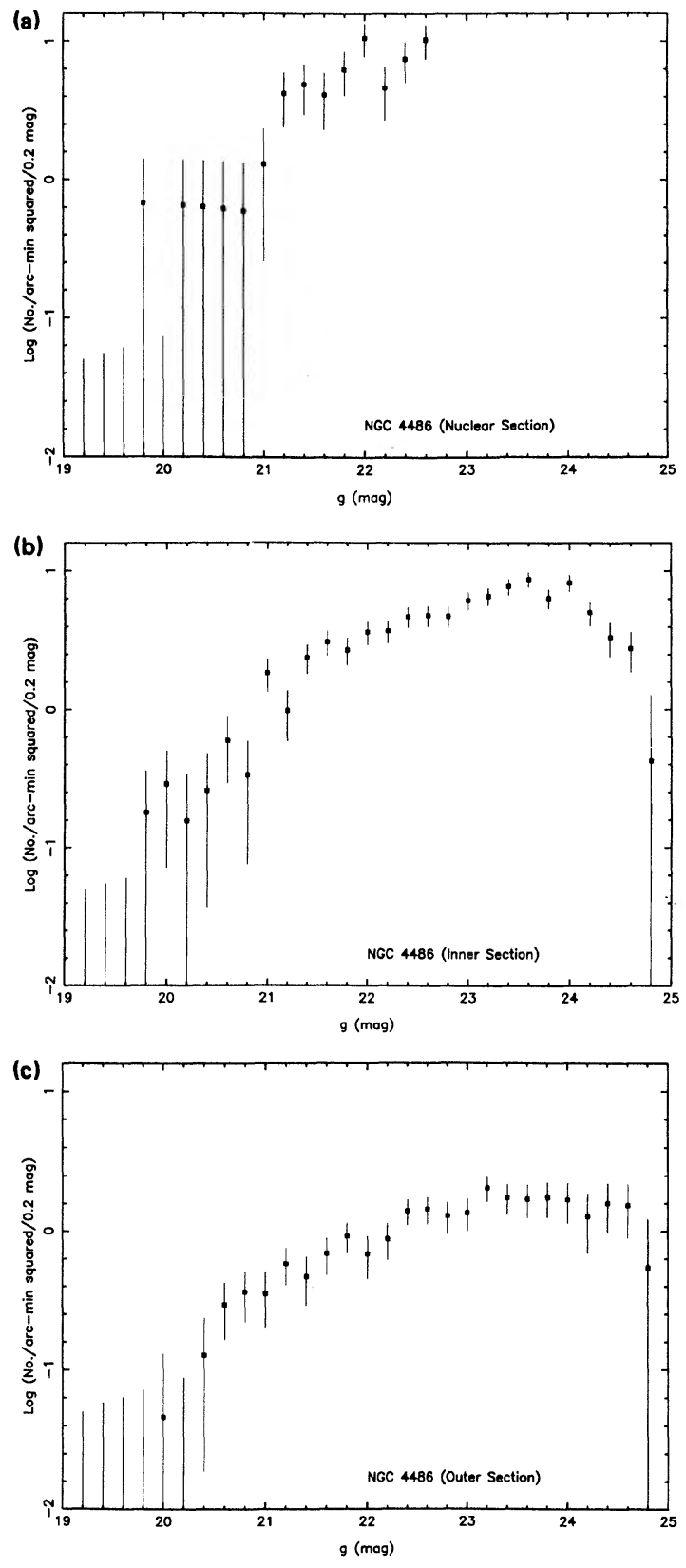

FIG. 4. Same as Fig. 3 for NGC 4486.

crowding corrections in the inner part of NGC 4486 are in fact even larger than the values we used (deduced from Table IV). The peak in the luminosity function is at $g \approx 24.2$, with a peak at $g<23.8$ being absolutely ruled out. This corresponds to $B \approx 24.85$, which is in good agreement with the value of $B=25.0 \pm 0.3$ adopted by van den Bergh, Pritchet, and Grillmair (1985) in their discussion of the distance to NGC 4486 using the luminosity function of its globular clusters as compared to that of globular clusters in Local Group galaxies. We thus support their distance determination to the Virgo cluster, if the commonly made assumption that there exists a unique functional form and peak magnitude for all globular cluster luminosity functions is, in fact, correct. While one can imagine attempting to derive a more accurate luminosity function for the globular clusters in the Virgo ellipticals at the faintest end, this would require frames where the crowding correction at $g \approx 25$ mag is smaller than in the present data. Superb seeing, high-quantum-efficiency detectors, and the utmost care in the details of the flatfield algorithm would be necessary.

Figure 5 shows a comparison of our background- and crowding-corrected $N_{c}$-magnitude distribution for the outer part of NGC 4486 (where the uncertainties for each point are shown as in Fig. 4(c) with the luminosity function of van den Bergh, Pritchet, and Grillmair (1985)). The latter values, indicated by light circles, have been arbitrarily scaled to account for the difference in the area surveyed and in the distance from the center of NGC 4486 of their field. The uncertainties of the van den Bergh, Pritchet, and Grillmair values are not shown, but they cannot be smaller than the uncertainties of the present data, since a significantly smaller area was used both for the background and the galaxy field, and the seeing on their summed frames was worse than on ours. The agreement between the two luminosity functions is acceptable given the uncertainties involved.

\section{THE COLORS OF THE CLUSTER SYSTEMS}

\section{a) The Colors and Their Uncertainties}

The colors of the clusters were determined for objects with $g \leqslant 23.0 \mathrm{mag}$. As there is only one $r$ and $i$ exposure for each galaxy, a fainter cutoff would lead to inclusion of more objects with unreliable colors. This is sufficiently bright that contamination by background galaxies is not a problem; essentially all galaxies have already been eliminated. However, the background due to galactic stars (or quasars) is still present. To avoid the effects of a few background objects with colors very discrepant from those of the globular clusters, the cluster colors are given as the median, and the first and third quartile, within bins of semimajor axis, for each of the three galaxies in Table VI. Also listed for comparison are the colors of the light from the underlying halo of the galaxies themselves taken from Paper I. The nuclear subsets are included only in the $g-r$ colors, not in the $g-i$ color distributions. This is because the brighter sky and brighter galaxy background at $i$ conspire to saturate the frames at a distance correspondingly further from the nucleus. The reader should also note that the zero point of the $(g-i)$ colors for

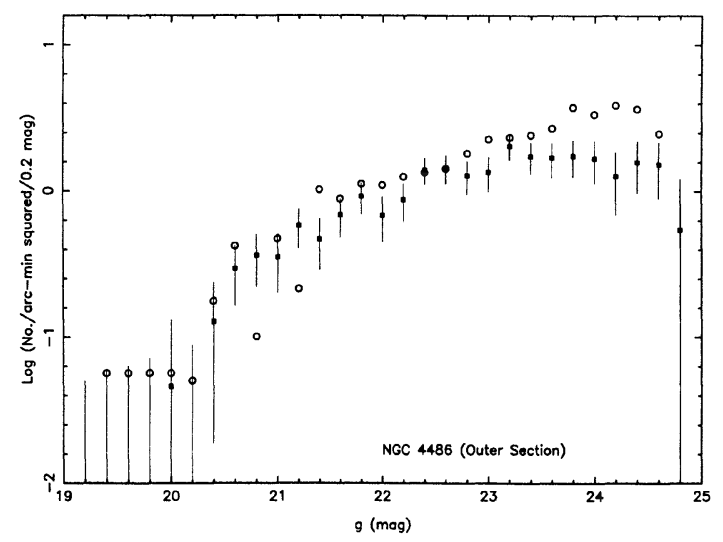

FIG. 5. The surface density of globular clusters in the outer part of NGC 4486 is indicated by the dark squares, which are identical to those of Fig. 4(c). The uncertainties are shown by the vertical lines. The light circles denote the surface densities found by van den Bergh, Pritchet, and Grillmair (1985). 


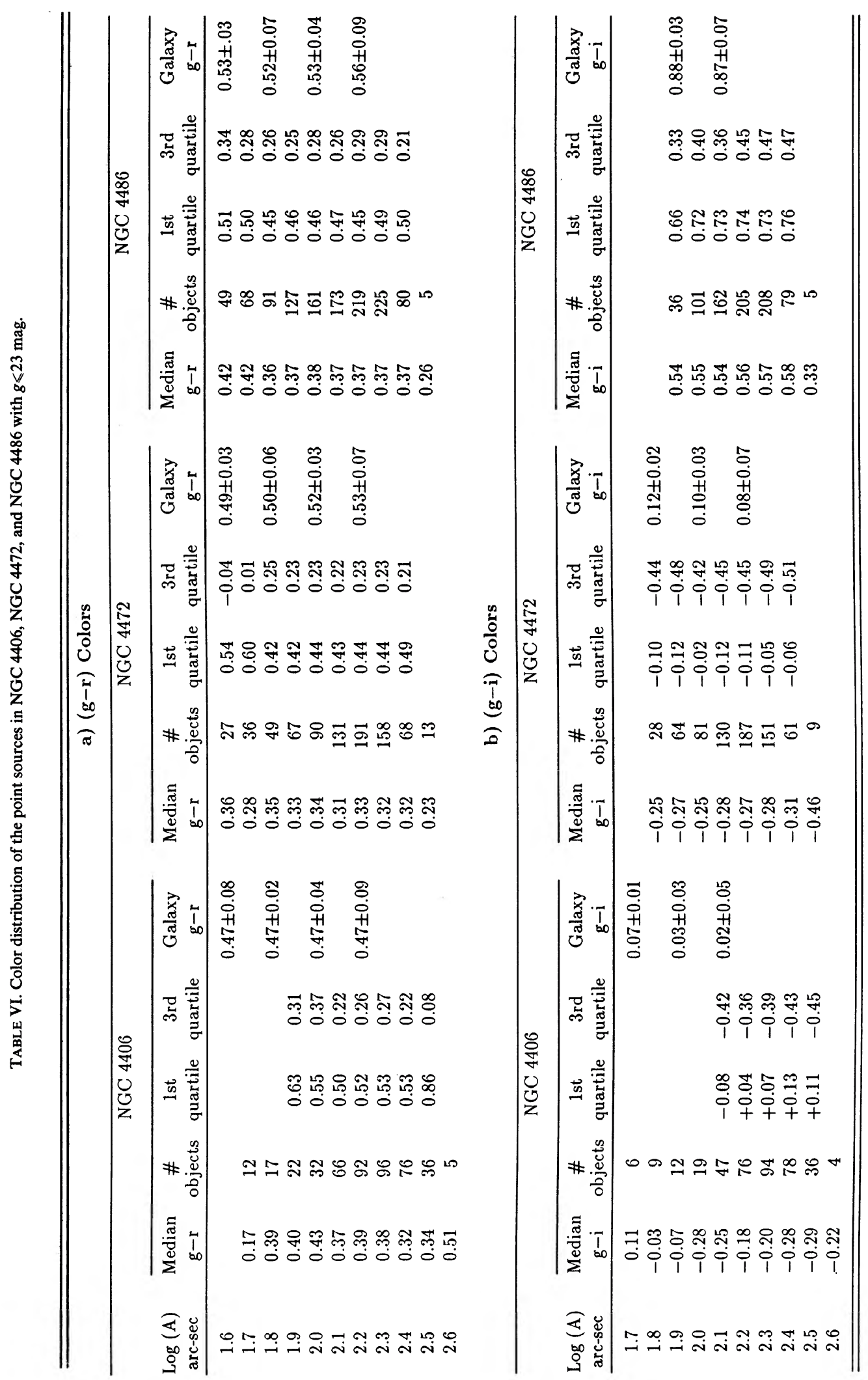


NGC 4406 and for NGC 4472 is the same arbitrary value used in Paper I.

The uncertainty in these colors can be deduced by adding stellar images to the frames as described in Sec. IV $b$, recovering them, and measuring the magnitudes of the artificial stars. This experiment was carried out at two magnitude levels: first at the cutoff in $g$ magnitude ( $g=23.0 \mathrm{mag}$ ), and then $0.5 \mathrm{mag}$ brighter $(g=22.5 \mathrm{mag})$. In each case the $r$ and $i$ limits were set so as to correspond to the mean color of the clusters. Table VII summarizes the results for NGC 4406 and NGC 4486, where typical trials involved about 300 stars in the outer section of the frames and about 45 stars in the inner section for each color. The table contains the rms deviation of the color of a single cluster about the mean, for those artificial stars that were recovered (i.e., those whose images do not by chance fall on top of a real object in the frame). (Note that in all cases more than $90 \%$ of the artificial stars were recovered.)

The first point to note from Table VI is that there is no obvious gradient in the median color of the globular clusters as one moves away from the nucleus of the galaxy. Any gradient in either $(g-r)$ or $(g-i)$ color is smaller than 0.1 mag over a factor of more than 5 in semimajor axis. As discussed in Paper I, the gradient in the color of the light of the underlying galaxy is also small, but definitely detectable; we find (from Table VI of Paper I) gradients of 0.05 mag in $(g-r)$ color and $0.07 \mathrm{mag}$ in $(g-i)$ color over the same spatial range. Such small gradients cannot be reliably detected in the data available for the globular cluster systems in Table VI. Furthermore, the distribution in color (i.e., the color at the first and third quartiles) within the globular cluster system also is independent of spatial position.

Table VI serves to verify one of the principal results found by Strom et al. (1981) and by Forte, Strom, and Strom (1981), namely that at any point the median of the colors of the globular clusters is bluer than that of the halo light of NGC 4486 itself. This result is now extended to all three Virgo ellipticals discussed here. The difference in $(g-r)$ color is $0.18 \pm 0.05 \mathrm{mag}$, with the difference in NGC 4406 possibly being somewhat smaller. This difference corresponds to a $4 \sigma$ to $6 \sigma$ result for each individual spatial bin, taking into account the uncertainties in the globular cluster

TABLE VII. Uncertainty of globular cluster colors based on distribution of colors of added point sources.

\begin{tabular}{lcccc}
\hline \hline & $\begin{array}{c}\text { NGC 4406 } \\
\sigma^{3}(g-r) \\
(\mathrm{mag})\end{array}$ & $\begin{array}{c}\text { NGC 4486 } \\
\sigma(g-r) \\
(\mathrm{mag})\end{array}$ & $\begin{array}{c}\text { NGC 4406 } \\
\sigma(g-i) \\
(\mathrm{mag})\end{array}$ & $\begin{array}{c}\text { NGC 4486 } \\
\sigma(g-i) \\
(\mathrm{mag})\end{array}$ \\
\hline$g=23.0$ & & & & \\
Inner $^{1}$ & 0.29 & 0.30 & 0.28 & 0.32 \\
Outer $^{2}$ & 0.24 & 0.29 & 0.28 & 0.35 \\
& & & & \\
$g=22.5$ & & & & \\
Inner & 0.16 & 0.25 & 0.20 & 0.33 \\
Outer & 0.14 & 0.27 & 0.18 & 0.27 \\
\hline \hline
\end{tabular}

Notes to TABLE VII

1. Inner clusters have $60 \leqslant A \leqslant 130$ arcsec.

2. Outer clusters have $A>130$ arcsec.

3. This is the $\sigma$ of the individual measurements about the mean for the recovered sources added to the frames. colors from Table VII, the number of clusters (from Table VI) and the uncertainty in the color of the galaxy light from Paper I repeated in Table VI. Our confidence in the reality of the color difference between the clusters and halo light for those three galaxies is very high.

We next examine the dependence of color on magnitude, considering only the case of NGC 4486, as it has the richest system of globular clusters. Figure 6 shows the $g$ magnitude versus the $(g-r)$ color for globular clusters in the inner subsets of NGC 4486 with $40 \leqslant A \leqslant 224$ arcsec. This spatial regime was chosen to avoid regions where the globular clusters do not dominate over the background field and to avoid the nucleus, where the colors are more uncertain. Table VIII presents the median and quartiles of the colors of these globular clusters in bins of $g$ magnitude 0.5 mag wide from $g=20.0$ to $23.0 \mathrm{mag}$. Again, there is no strong trend in median color with magnitude. The broadening out of the distribution toward fainter magnitudes is concentrated in the last magnitude $(22 \leqslant g \leqslant 23 \mathrm{mag})$ in the $(g-r)$ color and is larger in the $(g-i)$ color than in the $(g-r)$ color. The intrinsic distribution is that of the brighter clusters, while the broadening of the distribution at the faintest luminosities seen in both Fig. 6 and Table VIII is most likely due to the increasing uncertainties in the $r$ and $i$ magnitudes towards fainter luminosities given in Table VII.

\section{b) Comparison with the System of Galactic Globular Clusters}

We next compare these properties of the three Virgo globular cluster systems with those of the galactic globular clusters. From a very limited sample of data on the brightest globular clusters in NGC 4486, Ables, Newell, and O'Neill (1974) suggested that their mean $B-V$ color $(0.79 \mathrm{mag}$ ) was $\approx 0.1$ mag redder than the mean color of the sample of Milky Way globulars $\left(\langle B-V\rangle_{0}\right.$ of $\left.0.70 \mathrm{mag}\right)$. On the other hand, Hanes (1977) suggested that the color distributions of the two globular cluster systems were, in fact, identical. Our data provide a much more reliable answer to this question. For the galactic globular clusters, we use the metallicities of Zinn and West (1984) to derive the median $[\mathrm{Fe} / \mathrm{H}]$ of $-1.4 \mathrm{dex}$, with first and third quartiles at -0.85 and -1.75 dex. Since the majority of the as yet undiscovered galactic globular clusters must be near the galactic center, this is a representative distribution of the galactic globular cluster system as a whole outside the region within $0.5 \mathrm{kpc}$ of the center. (The equivalent area in the Virgo ellipticals is

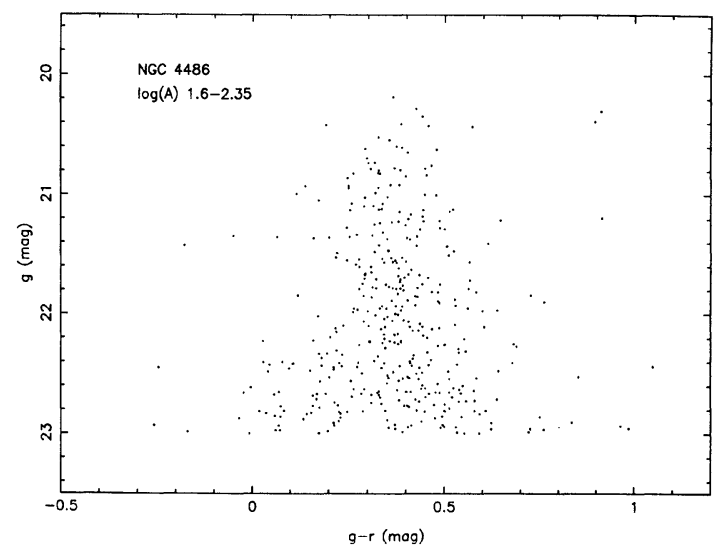

Fig. 6. The $g-r$ color as a function of $g$ luminosity for point sources in the field of NGC 4486 with $40 \leqslant A \leqslant 224$ arcsec. 
TABLE VIII. Colors as a function of luminosity for M87 globular clusters with $40 \leqslant A \leqslant 224$ arcsec.

\begin{tabular}{|c|c|c|c|c|c|c|c|c|}
\hline \multirow[b]{2}{*}{$\underset{(\mathrm{mag})}{g}$} & \multicolumn{2}{|c|}{$g-r$} & \multirow{2}{*}{$\begin{array}{c}\text { 1st } \\
\begin{array}{c}\text { Quartile } \\
\text { (mag) }\end{array}\end{array}$} & \multirow{2}{*}{$\begin{array}{c}\text { 3rd } \\
\text { Quartile } \\
\text { (mag) }\end{array}$} & \multicolumn{2}{|c|}{$g-i$} & \multirow{2}{*}{$\begin{array}{c}\text { 1st } \\
\begin{array}{c}\text { Quartile } \\
\text { (mag) }\end{array}\end{array}$} & \multirow{2}{*}{$\begin{array}{c}\text { 3rd } \\
\begin{array}{c}\text { Quartile } \\
\text { (mag) }\end{array}\end{array}$} \\
\hline & $\begin{array}{c}\text { Median } \\
\text { (mag) }\end{array}$ & No. & & & $\begin{array}{c}\text { Median } \\
\text { (mag) }\end{array}$ & No. & & \\
\hline $20.0-20.5$ & 0.44 & 9 & & & 0.60 & 9 & & \\
\hline $20.5-21.0$ & 0.33 & 32 & 0.39 & 0.30 & 0.48 & 32 & 0.55 & 0.39 \\
\hline $21.0-21.5$ & 0.35 & 53 & 0.44 & 0.29 & 0.53 & 51 & 0.65 & 0.45 \\
\hline $21.5-22.0$ & 0.38 & 82 & 0.46 & 0.32 & 0.54 & 77 & 0.70 & 0.44 \\
\hline $22.0-22.5$ & 0.37 & 100 & 0.45 & 0.24 & 0.55 & 89 & 0.71 & 0.37 \\
\hline $22.5-23.0$ & 0.40 & 137 & 0.51 & 0.21 & 0.63 & 117 & 0.81 & 0.39 \\
\hline
\end{tabular}

always excluded in the present study as the frames are saturated there.) Using published $B-V$ photometry of the integrated light of galactic globular clusters, we deduce the difference in $B-V$ color between the first and third quartiles of the galactic globular cluster system to be about $0.15-0.20$ mag, which corresponds to about the same difference in the $(g-r)$ color using the transformation equations of Cohen (1985). However, when we transform the median $(g-r)$ color for the NGC 4486 globular clusters of 0.37 mag to $B-V$ we obtain $0.81 \mathrm{mag}$, which is redder than that of the majority of galactic globulars, even allowing for up to 0.04 mag in foreground galactic reddening as inferred by Burstein and Heiles (1984) from $21 \mathrm{~cm}$ neutral-hydrogen maps. We thus conclude that the median metallicity of the globular clusters of NGC 4486 may be $\approx 0.5$ dex higher than that of the galactic globular clusters. The same appears to be true for the two other galaxies, with the data being least reliable for NGC 4406, due to the sparsity of its globular clusters. A better estimate of the difference in mean metallicity between the globular cluster systems of the Milky Way and the Virgo giant ellipticals will only be possible when population-synthesis models of the integrated light of old stellar systems, such as that of Aaronson et al. (1978), are updated and expanded to other photometric systems.

\section{THE SPATIAL DISTRIBUTION OF THE CLUSTERS}

\section{a) The Shape of the Globular Cluster Systems}

We must first establish whether the spatial distribution of the globular clusters around each of the Virgo ellipticals follows the galaxy contours in ellipticity or has a different shape from the underlying galaxy. This is an extremely difficult question to answer if the number of clusters is not large. We assume initially that the position angle of the major axis of each of the giant ellipticals is independent of distance from the nucleus. Although the results of Paper I demonstrate that there is some twist to the galaxy isophotes, particularly in NGC 4472, this is an acceptable approximation to the level of accuracy required here. We begin by deriving the distribution of point sources around each galaxy with respect to the sector angle $(S)$, where that is defined as the position angle of a source with respect to the major axis of the galaxy. Thus the major axis of the galaxy corresponds to $S=0$, or $180^{\circ}$, while the minor axis has $S=90$, or $270^{\circ}$. We choose an interval in radius and in magnitude over which the globular clusters dominate the point-source distribution, rather than the background dominating. The sector angle is then calculated for each point source on the master lists that meets the radius and magnitude limits and a histogram is formed over twelve $30^{\circ}$ bins in $S$, where the first section is $-15 \leqslant S \leqslant 15^{\circ}$ and includes the major axis. We also calculate the area actually searched in each bin of $S$, with the appropriate windows, guider arm, etc., excluded. We can then determine the surface density of point sources, corrected for the background in each of the 12 bins in $S$. These results for the three Virgo ellipticals are given in Table IX, where the $\sigma$ is that of Poisson statistics for the counts of the sources in each bin. The histogram in $S$ is plotted for NGC 4486 in Fig. 7. An illustrative fit, $N=34.1+8 \cos (2 S)$, is shown to guide the eye. For NGC 4486, the answer is clear; the globular cluster system is not spherical, and it has the same position angle (to within $\pm 20^{\circ}$ ) as does the galaxy. Furthermore, if we assume that the ellipticity $(\epsilon)$ is the same for the galaxy and the globular clusters, we can predict what the ratio of surface densities along the major and minor axis of NGC 4486 should be. One expects this ratio to be $(1-\epsilon)^{E}$, where $E$ is the exponent in the power law for the surface density of globular clusters as a function of semimajor axis. The value of $\epsilon$ for NGC 4486 in the spatial regime under consideration (taken from Paper I) is $0.15-0.20$, while the value of $E$ is discussed later in this section and is listed in Table X. Adopting $E=-1.5$, we predict the surface density ratio of globular clusters between the major and minor axis of NGC 4486 to be $0.72-0.78$. Figure 7 and Table IX show that reasonable agreement exists between this prediction and the observed ratio. Therefore, the shape of the isophotes and the shape of the globular cluster distribution in NGC 4486 are identical. This conclusion is in agreement with that of Harris and Smith (1976).

The situation is more difficult for the two other galaxies because of the lower surface density of globular clusters compared to the background. For NGC 4406, the globular cluster system is so sparse that we had to reflect the histogram in bins in $S$ about the minor axis (thus cutting the number of intervals from twelve to six) in order to obtain the result listed in Table IX. This table shows that the surface density (with the background subtracted) has a minimum in the two bins in $S$ corresponding to the minor axis of each galaxy. Thus we believe that in both cases the globular cluster systems are elliptical rather than spherical, and their position angles appear to be close to those of the underlying galaxies. However, the statistics are too poor to allow a realistic determination of the value of $\epsilon$ itself.

Harris and Petrie (1978) believed that the globular clus- 
TABLE IX. Eccentricity of the globular cluster systems in NGC 4406, NGC 4472, and NGC 4486.

\begin{tabular}{|c|c|c|c|c|c|c|}
\hline \multirow[b]{2}{*}{$\begin{array}{l}\text { Sector Angle } \\
\quad \text { (degrees) }\end{array}$} & \multicolumn{2}{|c|}{ NGC $4406^{2}$} & \multicolumn{2}{|c|}{ NGC $4472^{3}$} & \multicolumn{2}{|l|}{ NGC $4486^{4}$} \\
\hline & $\begin{array}{c}\mathrm{N} \\
\left(\mathrm{No} / \mathrm{arc}-\mathrm{min}^{2}\right)\end{array}$ & $\sigma$ & $\begin{array}{c}\mathrm{N} \\
\left(\text { No. } / \operatorname{arc}-\min ^{2}\right)\end{array}$ & $\sigma$ & $\begin{array}{c}\mathrm{N} \\
(\text { No./arc-min } \\
\end{array}$ & $\sigma$ \\
\hline-15 to 15 & 18.9 & 4.3 & 43.7 & 7.7 & 43.2 & 3.2 \\
\hline 15 to 45 & 19.9 & 4.2 & 30.8 & 6.2 & 35.0 & 3.0 \\
\hline 45 to 75 & 9.2 & 2.4 & 42.4 & 9.7 & 32.9 & 2.6 \\
\hline 75 to 105 & 9.0 & 2.9 & 16.2 & 5.4 & 28.5 & 2.4 \\
\hline 105 to 135 & 21.4 & 5.0 & 24.4 & 7.7 & 37.6 & 3.2 \\
\hline 135 to 165 & 13.4 & 3.5 & 40.6 & 9.3 & 37.8 & 2.9 \\
\hline 165 to 195 & & & 34.4 & 7.2 & 38.2 & 3.0 \\
\hline 195 to 225 & & & 33.2 & 6.3 & 27.3 & 2.6 \\
\hline 225 to 255 & & & 39.2 & 6.9 & 28.6 & 2.8 \\
\hline 255 to 285 & & & 21.1 & 4.7 & 21.0 & 2.1 \\
\hline 285 to 315 & & & 18.1 & 6.8 & 36.1 & 3.6 \\
\hline 315 to 345 & & & 26.1 & 5.7 & 42.8 & 3.2 \\
\hline
\end{tabular}

\section{NOTES}

1. The Sector Angle is measured from the major axis of the galaxy.

2. For 90 point sources with $21.0 \leq g \leq 23.5 \mathrm{mag}$ and $25 \leq R \leq 80$ arc-sec. The results have been reflected about the minor axis.

3. For 245 point sources with $21.0 \leq g \leq 23.5 \mathrm{mag}$ and $30 \leq R \leq 100$ arc-sec.

4. For 1678 point sources with $21.0 \leq g \leq 24.0 \mathrm{mag}$ and $70 \leq R \leq 224$ arc-sec.

ter system of NGC 4472 is spherical. However, the predicted ratio of the major- to minor-axis surface density is smaller than they would have expected, because, as described subsequently, the globular cluster system is more extended than the light of the underlying galaxy. This decreases the apparent asymmetry in the globular cluster system. In addition, Harris and Petrie used counts over a very large radius regime in NGC 4472 extending out to 10 arcmin (for the innermost of the two annuli they considered), and the large background (which was not removed in their figure) makes it difficult to see any position-angle dependence.

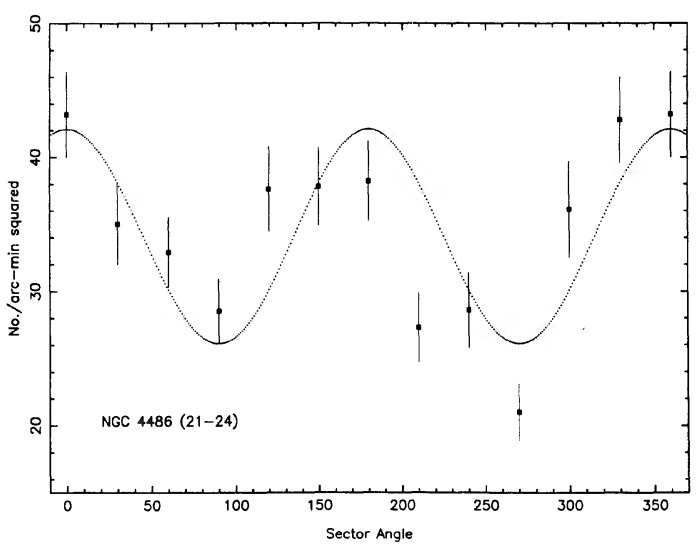

Fig. 7. The background-corrected count rate of point sources per $\operatorname{arcmin}^{2}$ in NGC 4486 with $21 \leqslant g \leqslant 24 \mathrm{mag}$, and $70 \leqslant R \leqslant 224 \mathrm{arcsec}$, where $R$ is the radius from the center of the galaxy, is plotted as a function of sector angle. The dashed line represents an illustrative fit $(N=34.1+8.0 \cos (2 S))$.

\section{b) The Distribution with Semimajor Axis}

We continue by explicitly assuming, based on the results of the previous section, that the surface density of the globular clusters in each of the three galaxies has the same shape as the isophotes of each galaxy. We can thus calculate the spatial position in semimajor axis $(A)$ with respect to the center of the galaxy for each of the detected point sources using the ellipse fits for the Virgo ellipticals from Paper I. (These include $\epsilon$ and galaxy position-angle function varying with semimajor axis.) In order to calculate the spatial distribution of the globular clusters in $A$, we need also the background density (number in a given magnitude interval per $\operatorname{arcmin}^{2}$ ), and the total area within the MASTERG frames that was searched for point sources in each semimajor-axis bin. The area searched was found by calculating the semimajor axis for each pixel in each of the four chips of the MASTERG frames, blanking out the excluded areas (windows, guider arm, etc.), and summing over bins in the logarithm of $A$. Figures $8(\mathrm{a})-8(\mathrm{c})$ give the results for the three galaxies, namely the number/ $\operatorname{arcmin}^{2}$ of detected point sources within the magnitude interval $21 \leqslant g \leqslant 24$, corrected for the summed background source density within the same magnitude interval. Let us denote the number of clusters/arcmin ${ }^{2}$ in this magnitude interval, corrected for the background, as $N(21-24)$.

The increment in $\log (A)$ in Figs. 8(a) $-8(\mathrm{c})$ is 0.03 dex. In the central regions, the area corresponding to such a bin in $A$ is very small, and some intervals in $\log (A)$ have no detected stars; these are indicated by light circles, which are plotted as though there were two detected globular clusters in the spatial interval within the relevant magnitude range. The last few points with the largest $A$ values plotted in Figs. 
TABLE X. Spatial distribution parameters.

\begin{tabular}{ccccc}
\hline \hline Galaxy & $\begin{array}{c}\mathrm{g} \\
\text { Interval } \\
(\mathrm{mag})\end{array}$ & $\begin{array}{c}\mathrm{Log}\left(\mathrm{N} / \mathrm{arc-min}^{2}\right) \\
\text { at } A=100 \text { arc-sec }^{-}\end{array}$ & $\begin{array}{c}\mathrm{E} \\
\text { (cluster system) }\end{array}$ & $\begin{array}{c}\mathrm{E} \\
\text { (galaxy light) }\end{array}$ \\
\hline \multirow{2}{*}{ NGC 4406 } & $21-23$ & 0.70 & $-1.68 \pm 0.15$ & -1.50 \\
& $21-24$ & 1.08 & $-1.48 \pm 0.10$ & \\
NGC 4472 & $21-23$ & 1.15 & $-1.00 \pm 0.15$ & -1.60 \\
& $21-24$ & 1.40 & $-1.18 \pm 0.10$ & \\
NGC 4486 & $21-23$ & 1.45 & $-1.45 \pm 0.10$ & -1.80 \\
& $21-24$ & 1.75 & $-1.50 \pm 0.15$ & \\
\hline \hline
\end{tabular}

$8(a)-8$ (c) also have relatively small areas as only a small segment of the full annulus in $A$ is sampled, that corresponding to the appropriate corners of the MASTERG frames.

It is interesting to compare the spatial profiles of the cluster distributions among the three galaxies and also with the profile of the integrated light of the underlying galaxy taken from Paper I. There have been many suggestions, beginning with that of Harris and Racine (1979), that the globular cluster system in NGC 4486 has a larger effective radius than the light from the spheroid of the galaxy. Again, our data can provide a definitive answer to this important question. Figures $8(a)-8(c)$ indicate that in the outer parts of the galaxies, in each case the number density- $A$ distribution can be approximated by a power law. The exponent $E$ of the relationship $N(21-24)=N_{0} \times A^{E}$ is tabulated for each galaxy in Table $X$. The same quantity can be derived from the integrated light profile in the $g$ filter bandpass for the underlying galactic halo; $E$ (galaxy) for $A \approx 100$ arcsec is also listed in the table. The $g$ intensity profile for the galaxy (divided by a constant) is shown as the solid line in Figs. 8(a)-8(c). The constant is identical for each of the three Virgo ellipticals. It is clear that for NGC 4406, the globular cluster spatial distribution and that of the underlying galaxy are identical to within the uncertainties of each. However, for both NGC 4472 and NGC 4486, the globular cluster distribution is more extended than in the underlying galactic light. Comparison of the figures also shows clearly the relative excess of globular clusters per unit galaxy luminosity in NGC 4486.

One may examine whether this holds even into the centers of the galaxies. Although our data on the spatial distribution of the globular clusters do not extend inward of $A=25$ arcsec, we examine the interval in $A$ from 25 to 40 arcsec carefully. The relevant areas are small (and smallest in NGC 4406, which is the most eccentric of the three galaxies). However, note that within the corresponding interval in $\log (A)(1.4-1.6$ dex $)$ in Figs. 8(a)-8(c) there are several points that seem unexpectedly low and a few light circles (denoting no detected objects in the 0.03 dex interval in $\log (A))$. It is thus apparent that in M87 and in NGC 4472 the number density $(N(21-24))$ does not continue to rise as steeply for $A \leqslant 40$ arcsec, and may, in fact, be approximately constant within that interval. This extends the result of Lauer and Kormendy (1986) and of Grillmair, Pritchet, and van den Bergh (1986) for NGC 4486 to NGC 4472 as well. The statistics for NGC 4406 are not as good, and it is not clear whether the plateau in $N(21-24)$ is present.

We are also now in a position to compare the total number of clusters in the three galaxies. From the total count rate of point sources with $g \leqslant 24 \mathrm{mag}$ in the entire area search of the MASTERG frame of each galaxy, corrected for the density of background objects, we derive the ratio of the total number of globular clusters as 1.9 $( \pm 0.2): 1: 0.25( \pm 0.08)$ for NGC 4486, NGC 4472, and NGC 4406, respectively. These ratios agree with those derived from smoothed versions of the spatial distributions given in Figs. $8(\mathrm{a})-8(\mathrm{c})$. (Because the galaxies have different ellipticities and different centering on the 4-Shooter frame, the ratios of the total number of clusters out to $A=\infty$ may be slightly different.) We use the ratio of the total visual luminosity from the Second Reference Catalog of Bright Galaxies (de Vaucouleurs et al. 1976) to determine the ratios of the specific frequency of globular clusters $S$ defined by Harris and van den Bergh (1981) for the three galaxies to be 2.4:1:0.5 for NGC 4486, NGC 4472, and NGC 4406, respectively. Thus $S(N G C 4472)$ may be somewhat larger than for NGC 4406, while $S$ (NGC 4486) is indubitably anomalously large, as has been found by many previous investigators, including Hanes (1977), Harris and van den Bergh (1981), and Harris (1986). We must recall that all these statistics were calculated within the interval $21 \leqslant g \leqslant 24 \mathrm{mag}$, where the background corrections are relatively small and any uncertainty in the background can contribute only a negligible uncertainty to these results.

\section{SUMMARY AND IMPLICATIONS FOR GALAXY FORMATION \\ a) Summary of Major Results}

We summarize below the major results of our investigation of the globular cluster systems around three of the brighter ellipticals in the Virgo cluster of galaxies. Two of these are very luminous ellipticals (NGC 4472 and NGC 4486), while one is more ordinary in its luminosity. The principal advantages of our analysis compared to the many earlier studies of these systems are the use of a digital CCD detector, so that easier and more accurate subtraction of the halo light of the underlying galaxy can be achieved, and the use of a mosaic of CCD detectors (i.e., the 4-Shooter instrument), to enable simultaneous coverage of a large spatial 
field at good scale to a deep limiting magnitude. Throughout the paper, we have tried to indicate within each section how our results are in some cases completely new, and in other cases extend or confirm those found by the many previous analyses of these swarms of globular clusters.

As described above, all galaxies brighter than about 22.5 mag have been eliminated, but fainter than that magnitude many galaxies are too small to have been picked out as spa-
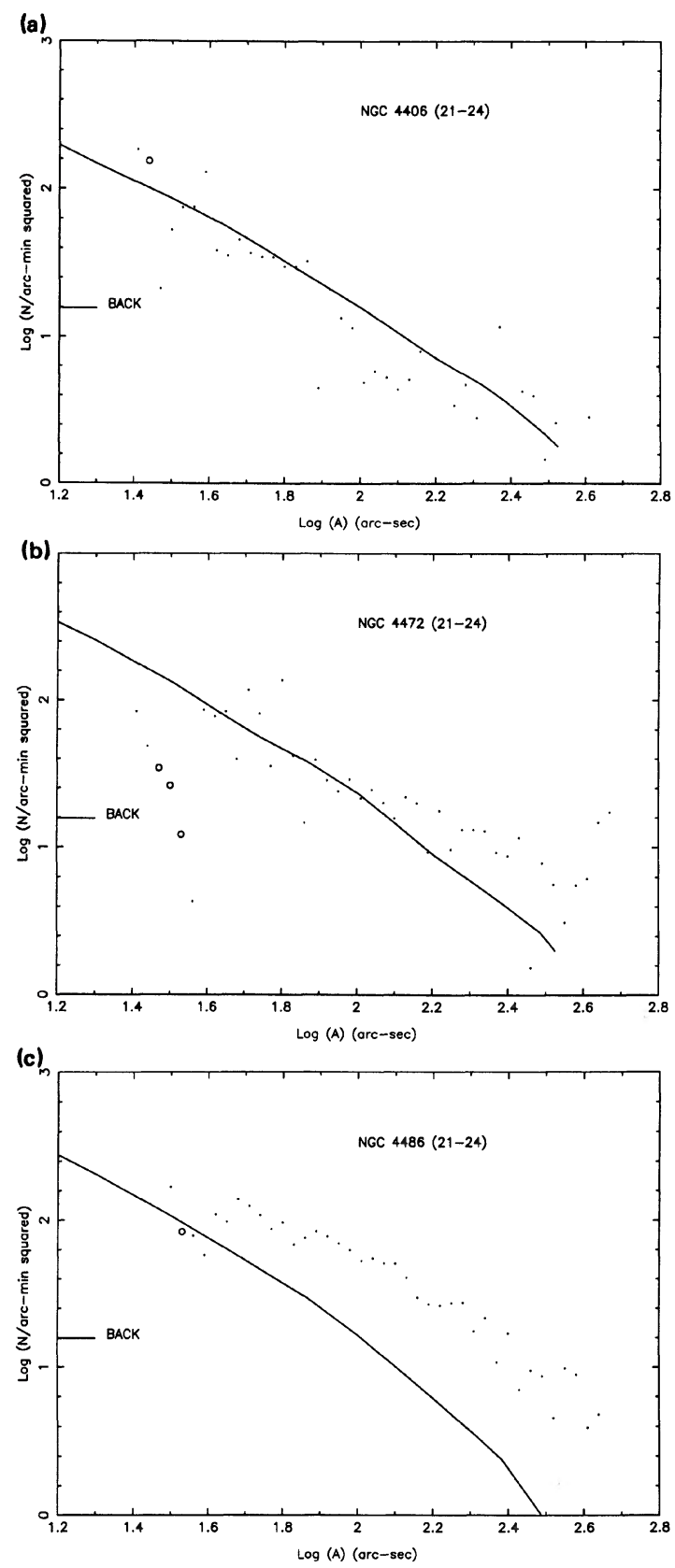

FIG. 8(a). The spatial distribution of the globular clusters in NGC 4406 with $21 \leqslant g \leqslant 24 \mathrm{mag}$ is shown as a function of the semimajor axis. The dots indicate the actual count rate with the background (whose value is indicated by the horizontal line) removed. The light circles indicate intervals in semimajor axis with no detected globular clusters which are plotted as though there were two detected globular clusters in the appropriate spatial interval with $21 \leqslant g \leqslant 24 \mathrm{mag}$. (b) Same as Fig. 7(a) for NGC 4472. (c) Same as Fig. 7(a) for NGC 4486. tially extended objects. The limiting magnitude we have attempted to work to is $g \approx 25.0$, but between $24.5 \leqslant g \leqslant 25.0$ both the completeness corrections and the magnitude uncertainty due to fluctuations in the background (consisting of light from the night sky plus the galaxy halo) increase rapidly. Because our frames go very deep, the nuclear regions of each of the three galaxies are saturated, and no identification of clusters closer than 25 arcsec to the nucleus is possible.

We find no detectable gradient in the median color of the globular clusters as one moves out in either radius or semimajor axis from the nucleus of each of three galaxies. This result complements that of Paper I, where the color gradient in the underlying halo light of the galaxy was found to be considerably smaller than in several previous studies. The median color of the globular clusters is at each point in the galaxy halo bluer than that of the integrated light of the underlying halo stellar population. As described in Sec. V, these statistical and photometric errors are sufficiently small that this color difference must be accepted as real in each of the three galaxies. For NGC 4486, the color distribution of the globular clusters is independent of position and luminosity (at least until measuring errors in the colors dominate the spread in color at $g \approx 22.5 \mathrm{mag}$ ). These last two points are less certain for NGC 4472, and the sparsity of the cluster system of NGC 4406 does not permit any discussion of these issues for that galaxy. The median color of the globular clusters in each of the three galaxies is definitely slightly redder than that for the system of globular clusters in the Milky Way. This color difference corresponds to a mean metallicity enhancement of about $0.5 \mathrm{dex}$ (for systems assumed to have the same age as the Milky Way globulars). (This statement too is less certain for NGC 4406 only.)

The globular cluster system has the same ellipticity and position as the halo light of the underlying galaxy. It is not a spherical distribution. (This result is definite for NGC 4486 and NGC 4472, and somewhat less certain for NGC 4406, but still quite suggestive.) The globular cluster system is more extended spatially than the light of the underlying galaxy in NGC 4486 and NGC 4472, but does not appear to be so in NGC 4406.

The magnitude distribution (histogram of number of clusters at a given magnitude) may be somewhat broader in NGC 4486 than it is in NGC 4472. In each case, the magnitude histogram appears to turn over at about $g \approx 24.2$ (not brighter than $g=23.8 \mathrm{mag}$ ). The best fit to the numbermagnitude histogram cannot be a power law, but must be some more complicated shape. For present purposes, we have adopted a Gaussian form to derive constraints on the distance to the Virgo cluster as described in Sec. IV $c$, but the exact form of the distribution fainter than $g \approx 24$ mag is poorly determined.

The total number of globular clusters per unit luminosity of the underlying galaxy halo light is abnormally large in NGC 4486.

\section{b) Implications for Galaxy Formation}

The formation of the system of globular clusters around a galaxy is intimately related to the formation of the galaxy itself. Among the best-developed scenarios are those of Fall and Rees (1985, 1987), where the globular clusters are the first objects formed as a galaxy collapses, and the halo is partially formed by the disruption of globular clusters themselves. The efficiency of disruption is undoubtedly a function of local density and hence of perigalactic distance of the orbit 
TABLE XI. Dwarf galaxies near three Virgo ellipticals.

\begin{tabular}{lccccccccl}
\hline \hline Galaxy & $\begin{array}{c}\Delta(x, y) \\
\text { arc-sec }\end{array}$ & $\begin{array}{c}\text { VCC } \\
\text { No. }\end{array}$ & $\begin{array}{c}\theta \\
(\mathrm{deg})\end{array}$ & $a / b$ & $\begin{array}{c}a_{\max } \\
(\operatorname{arc-sec})\end{array}$ & $g_{\text {tot }}$ & $g-r$ & $g-i$ & Remarks \\
\hline NGC 4406 & $-49,66$ & 882 & +50 & 1.4 & 12 & 15.44 & 0.50 & -0.22 & elliptical with its own globular clusters \\
NGC 4472 & $-275,267$ & 1254 & $\ldots$ & 1.1 & 22 & 15.26 & 0.48 & $\ldots$ & $\begin{array}{l}\text { elliptical with its own globular clusters } \\
\text { irregular, partly off frame }\end{array}$ \\
& $\approx-209, \approx-276$ & 1249 & $\ldots$ & $\ldots$ & $\ldots$ & $\ldots$ & $\ldots$ & $\ldots$ & approx. round elliptical \\
& $+144,-243$ & 1203 & $\ldots$ & 1.0 & 11 & 15.72 & 0.36 & $\ldots$ & approx. round elliptical \\
NGC 4486 & $+179,+215$ & 1199 & $\ldots$ & 1.0 & 9 & 15.82 & 0.54 & -0.02 & apprar \\
& $-205,-139$ & $\ldots$ & $\ldots$ & $\ldots$ & 12 & 20.77 & 0.23 & 0.21 & partially resolved irregular \\
\hline \hline
\end{tabular}

of the globular cluster, as discussed by Fall and Rees (1977) and by Ostriker (1987). De Young, Lind, and Strom (1983) discuss the possible chemical enrichment of halo gas by ejecta from stars in globular clusters, assuming that star formation in the galaxy halo occurs later than the formation of the globular clusters. The recent simulations of exchange of globular clusters among galaxies in rich clusters by Muzzio et al. (1984) and Muzzio (1986) provide an interesting new idea. One must also remember the possibility of mergers among the galaxies themselves, particularly in the core of a cluster as rich as the Virgo cluster (see, for example, Merritt 1984).

Our current understanding of the observational facts about extragalactic globular cluster systems and how they compare with the predictions of the above theoretical analyses has been reviewed by Harris (1986). In the present work, several relevant facts have emerged as definite conclusions, whereas earlier they had to be considered as suggestions applying in many cases only to the best-studied case of NGC 4486 with its extensive cluster system. The most important of these which must be predicted by any theory are the difference in mean color between the globular clusters and the halo at a given location, the greater spatial extent of the cluster systems, and the higher velocity dispersion found by Mould, Oke, and Nemec (1987) and by Huchra and Brodie (1987). Also important is the overall similarity in the properties of the globular cluster systems of NGC 4486 and NGC 4472 , in spite of the fact that one galaxy is located in the core of the Virgo cluster, and hence much more likely to accrete nearby galaxies or to exchange globular clusters with its neighbors, while the other is situated in a much less dense environment.
Progress in refining the theories can only be achieved by better observational studies of other galaxies with rich systems of globular clusters. This will require observations of even more distant clusters than Virgo, pushing groundbased instrumentation to its absolute limit, and may well be best pursued with the Hubble Space Telescope.

I gratefully acknowledge support from the Caltech Recycling Center and from Gaston Araya Machining. I am grateful to the referee for a very careful reading of the manuscript.

\section{APPENDIX: THE DWARF GALAXIES}

The properties of the dwarf galaxies that are located on our frames and appear to be associated with the Virgo cluster are tabulated below (Table XI). They are cross referenced to the catalog of galaxies in the Virgo cluster by Binggeli, Sandage, and Tammann (1985). At least two of the dwarf ellipticals appear to have globular cluster systems of their own. The photometric zero points are those of the present work adopted from Paper I. We have not attempted to derive information on the possible presence of color gradients for these small systems due to differences in seeing among the frames of various colors. The magnitude of VCC 882, the dwarf galaxy near NGC 4406, appears to be incorrect in the VCC catalog, which is not very surprising as this is the dwarf galaxy closest to the center of a large elliptical from the present list. The object near NGC 4486 is of particular interest. It is quite faint and partially resolved. The classifications, the radii, and the ellipticities are in quite good agreement between the present data and the VCC catalog.

\section{REFERENCES}

Aaronson, M., Cohen, J. G., Mould, J. R., and Malkan, M. (1978). Astrophys. J. 223, 824

Ables, H. D., Newell, E. F., and O'Neil, E. J., Jr. (1974). Publ. Astron. Soc. Pac. 86, 311.

Binggeli, B., Sandage, A., and Tammann, G. A. (1985). Astron. J.90, 1681. Burstein, D., and Heiles, C. (1984). Astrophys. J. Suppl. 54, 33.

Cohen, J. G. (1985). Astron J. 90, 2254.

Cohen, J. G. (1986). Astron J. 92, 1039 (Paper I).

de Vaucouleurs, G., de Vaucouleurs, A., and Corwin, H. G., Jr. (1976).

Second Reference Catalog of Bright Galaxies (University of Texas, Austin).

De Young, D. S., Lind, K., and Strom, S. E. (1983). Publ. Astron. Soc. Pac. 95, 401.

Fall, S. M., and Rees, M. J. (1977). Mon. Not. R. Astron. Soc. 181, 37P. Fall, S. M., and Rees, M. J. (1985). Astrophys. J. 298, 18.

Fall, S. M., and Rees, M. J. (1987). In Globular Cluster Systems in Galaxies, edited by J. E. Grindlay and A. G. D. Philip (Reidel, Dordrecht).

Forte, J. C., Strom, S. E., and Strom, K. M. (1981). Astrophys. J. Lett. 245, L9.

Grillmair, C., Pritchet, C., and van den Bergh, S. (1986). Astron. J. 91, 1328.

Hanes, D. A. (1977). Mon. Not. R. Astron. Soc. 179, 331.

Harris, W. E. (1986). Astron. J. 91, 822.

Harris, W. E., and Petrie, P. L. (1978). Astrophys. J. 223, 88.

Harris, W. E., and Racine, R. (1979). Annu. Rev. Astron. Astrophys. 17, 241.

Harris, W. E., and Smith, M. G. (1976). Astrophys. J. 207, 1036.

Harris, W. E., and Smith, M. G. (1981). Astron. J. 86, 90.

Harris, W. E., and van den Bergh, S. (1981). Astron. J. 86, 1627.

Huchra, J., and Brodie, J. (1984). Astrophys. J. 280, 547.

Huchra, J., and Brodie, J. (1987). Astron. J. 93, 779.

Jarvis, J. F., and Tyson, J. A. (1981). Astron. J. 86, 476. 
Kron, R. (1980). Astrophys. J. Suppl. 43, 305.

Lauer, T. R., and Kormendy, J. (1986). Astrophys. J. Lett. 303, L1.

Merritt, D. (1984). Astrophys. J. 276, 26.

Mould, J. R., Oke, J. B., and Nemec, J. M. (1987). Astron. J. 92, 53.

Muzzio, J. C. (1986). Astrophys. J. 301, 23.

Muzzio, J. C., Martinez, R. E., and Rabolli, M. (1984). Astrophys. J. 285, 7.

Ostriker, J. P. (1987). In Globular Cluster Systems in Galaxies, edited by J. E. Grindlay and A. G. D. Philip (Reidel, Dordrecht).
Racine, R. (1968). J. R. Astron. Soc. Can. 62, 367.

Racine, R. (1980). In Star Clusters, edited by J. E. Hesser (Reidel, Dordrecht), p. 369.

Strom, S. E., Forte, J. C., Harris, W. E., Strom, K. M., Wells, D. C., and Smith, M. G. (1981). Astrophys. J. 245, 416.

Thuan, T. X., and Gunn, J. E. (1976). Publ. Astron. Soc. Pac. 88, 543.

Tyson, J. A., and Jarvis, J. F. (1979). Astrophys. J. Lett. 230, L153.

van den Bergh, S., Pritchet, C., and Grillmair, C. (1985). Astron. J. 90, 595.

Zinn, R., and West, M. (1984). Astrophys. J. Suppl. 55, 45. 